\title{
Jackiw-Teitelboim model coupled to conformal matter in the semi-classical limit
}

\author{
Upamanyu Moitra, Sunil Kumar Sake, Sandip P. Trivedi and V. Vishal \\ Department of Theoretical Physics, Tata Institute of Fundamental Research, \\ Colaba, Mumbai 400005, India \\ E-mail: upamanyu@theory.tifr.res.in, sunil.sake@tifr.res.in, \\ sandip@theory.tifr.res.in, vishal@theory.tifr.res.in
}

ABSTRACT: We analyse the Jackiw-Teitelboim model of 2D gravity coupled to $N$ massless free scalar fields in the semi-classical limit. Two systems are studied which essentially differ in the boundary conditions that are imposed. We find that the thermodynamics has interesting differences. We also analyse the response to additional infalling matter which satisfies the null energy condition. The second law is shown to be valid in both systems for the generalised entropy which takes into account the entanglement across the event horizon due to the matter fields. Similarly we find that the generalised entropy increases along future Q-screens in both systems.

KeYwords: 2D Gravity, AdS-CFT Correspondence, Black Holes, Models of Quantum Gravity

ArXiv EPrint: 1908.08523 


\section{Contents}

1 Introduction 1

2 Basic setup 2

2.1 Vacuum solutions 3

2.2 Infalling matter 4

$\begin{array}{lll}2.3 & \text { Second law } & 7\end{array}$

$\begin{array}{lll}\text { 2.3.1 The apparent horizon } & 7\end{array}$

3 Semi-classical analysis: the $\chi$ system $\quad 8$

3.1 Thermodynamics $\quad 9$

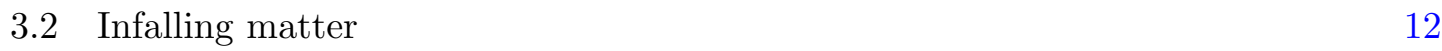

$\begin{array}{lll}3.2 .1 & \text { Some additional comments } & 14\end{array}$

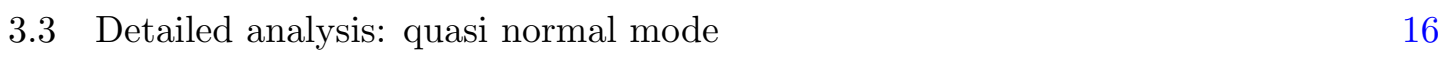

$\begin{array}{lll}3.4 & \text { Second law } & 19\end{array}$

4 Dynamical system with $\psi$ fields $\quad 21$

4.1 Entanglement entropy 22

4.2 Thermodynamics 23

4.3 Infalling matter 26

4.4 Second law 28

5 Generalised entropy and Q-screens $\quad 30$

5.1 The $\chi$ system 31

5.2 The $\psi$ system 32

6 Conclusions 33

$\begin{array}{ll}\text { A Coordinate transformations } & 35\end{array}$

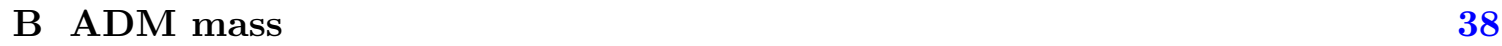

B.1 Fefferman-Graham coordinate transformation 38

$\begin{array}{lll}\text { B.2 Classical mass } & 39\end{array}$

B.3 Mass for the $\chi$ system $\quad 41$

B.4 Mass for $\psi$ system 42

C Late time behaviour of $h \quad 44$

C.1 Late time black hole coordinates 46

$\begin{array}{ll}\text { D Second law violation for apparent horizon } & 47\end{array}$ 


\section{Introduction}

The Jackiw-Teitelboim (JT) model [1, 2] of 2D gravity — see, for example, [3-5] for some early important work on the model - has attracted wide attention lately [6-54]. This model captures the behaviour of a class of statistical mechanics models called the SYK models [55-124] which have similarities with the behaviour of near-extremal black holes. In fact, it has been shown that the low-temperature and low-frequency behaviour of a wide class of near-extremal black holes, including rotating ones, is well approximated in a precise way by the JT model [9, 18, 30, 39, 48].

In this paper we consider the behaviour of $2 \mathrm{D}$ theories obtained by coupling the JT model to $N$ extra massless scalar fields, $\psi_{i}, i=1, \cdots N$. We work in the semi-classical limit obtained by taking $N \rightarrow \infty$ and the 2 D Newton's constant $G \rightarrow 0$, keeping $N G$ fixed. The quantum effects of matter are included in this approximation, while the gravity-dilaton sector behaves classically.

We examine two models here.

In the first model the effect of the $N$ scalars is replaced by one scalar called $\chi$ below, which is non-minimally coupled, with an action

$$
I_{\chi}=-\frac{N}{24 \pi} \int \sqrt{-g} \mathrm{~d}^{2} x\left[(\partial \chi)^{2}+R \chi\right]
$$

(and an appropriate boundary term, see eq. (3.3)). Due to the non-minimal coupling this single field reproduces the conformal anomaly of the $\psi_{i}$ fields originally present. The prefactor in eq. (1.1) scaling like $N$ also means that the field $\chi$ is classical in the large $N$ limit. The dynamics of the full system is then obtained by coupling this classical field to the classical gravity-dilaton system. The spacetime we consider has a boundary where the dilaton takes a fixed value; we also impose vanishing boundary conditions on $\chi$ at this boundary.

In the second model we work directly with the $N$ scalar fields $\psi_{i}, i=1, \cdots N$ which are minimally coupled with an action

$$
I_{\psi}=\frac{1}{2} \int \sqrt{-g} \mathrm{~d}^{2} x \sum_{i=1}^{N}\left(\partial \psi_{i}\right)^{2},
$$

and impose Dirichlet boundary conditions on these scalars.

We find that the two models have interesting differences. In the first model we impose Dirichlet boundary conditions on the $\chi$ field and in the second model Dirichlet boundary conditions on the $\psi_{i}$ fields; these are inequivalent. ${ }^{1}$

In both cases, infalling matter results in the formation of a black hole which evaporates and eventually settles down to thermal equilibrium. In this equilibrium state, matter is radiated by the black hole, bounces off the boundary and eventually falls back into the black hole, with the rate of Hawking evaporation equalling that of the infalling matter energy-momentum.

\footnotetext{
${ }^{1}$ It is not clear to us in fact if any allowed general boundary conditions imposed on the $\psi_{i}$ fields can lead to the same dynamics as that obtained by imposing Dirichlet boundary condition on the $\chi$ field.
} 
Quantum corrections result in corrections to the free energy and mass of the black hole as a function of temperature; these are different for the two systems. The first law of thermodynamics is obeyed in the presence of these corrections once the entropy is replaced by the generalised entropy which also includes a contribution coming from entanglement across the horizon.

For time-dependent situations, we find that once the external sources are turned off, the system relaxes to thermal equilibrium. In the $\chi$ system this relaxation is due to a quasi-normal mode which decays exponentially with an exponent which depends on the temperature $T$ and $G N$. In the $\psi$ system the system relaxes instantaneously.

Finally, we find that in both cases the Second Law of Thermodynamics is obeyed for the generalised entropy in the presence of additional classical matter which satisfies the null energy condition. We also analyse the behaviour of future Q-screens, which are an analogue of the locus swept out by the apparent horizon and find that the generalised entropy increases along the future Q-screen in both models. See, for instance, [125-137], and the references therein for some of the relevant literature.

This paper is structured as follows. In section 2 we review the classical behaviour of the JT model, including the black hole solution and the response to general infalling matter. The behaviour of the $\chi$ and $\psi$ systems is considered in section 3 and 4 respectively. The results of entropy monotonicity along Q-screen are discussed in section 5. Finally we end with conclusions in section 6. Appendices A-D contain important additional details.

\section{Basic setup}

The Jackiw-Teitelboim (JT) model consists of 2D gravity coupled to a scalar, $\phi$, called the dilaton, with an action,

$$
I_{J T}=\frac{1}{16 \pi G}\left(\int d^{2} x \sqrt{-g} \phi\left(R-\Lambda_{2}\right)+2 \int_{\mathrm{bdy}} \sqrt{-\gamma} \phi K-\frac{2}{L_{2}} \int_{\mathrm{bdy}} \sqrt{-\gamma} \phi\right) .
$$

Here $\Lambda_{2}$ is the $2 \mathrm{D}$ cosmological constant given by $\Lambda_{2}=-\frac{2}{L_{2}^{2}}$. The last term in the eq. (2.1) is a counter-term which is required to remove divergences that arise while computing the onshell action and related quantities. We shall set the $A d S_{2}$ length $L_{2}=1$ in the calculations to follow. Let us work in the conformal gauge in which the metric takes the form,

$$
\mathrm{d} s^{2}=-e^{2 \omega\left(x^{+}, x^{-}\right)} \mathrm{d} x^{+} \mathrm{d} x^{-} .
$$

The equation of motion by varying the dilaton in the above action is

$$
4 \partial_{+} \partial_{-} \omega+e^{2 \omega}=0 .
$$

It is easy to see that the equation above has a solution in which the spacetime is $A d S_{2}$, with the metric in Poincaré coordinates $(t, z)$ (related to the $x^{ \pm}$coordinates as $x^{ \pm}=t \pm z$ ) given by

$$
\mathrm{d} s^{2}=\frac{1}{z^{2}}\left(-\mathrm{d} t^{2}+\mathrm{d} z^{2}\right)=-\frac{4}{\left(x^{+}-x^{-}\right)^{2}} \mathrm{~d} x^{+} \mathrm{d} x^{-}
$$


Varying the metric we obtain,

$$
\frac{1}{8 \pi G}\left(\nabla_{\mu} \nabla_{\nu} \phi-g_{\mu \nu} \nabla^{2} \phi+g_{\mu \nu} \phi\right)=0
$$

This admits, as one of the solutions, a linearly varying dilaton

$$
\phi=\frac{1}{2 \mathcal{J} z},
$$

where $\mathcal{J}$ is an energy scale. The linear variation of the dilaton breaks the $\operatorname{SL}(2, R)$ isometries of $A d S_{2}$ to $\mathrm{U}(1)[9]$ and $\mathcal{J}$ characterises the scale of this breaking.

The spacetime has a boundary where the dilaton takes a fixed value

$$
\phi=\phi_{B}
$$

\subsection{Vacuum solutions}

The general solution for the dilaton satisfying eq. (2.5) is

$$
\phi=\frac{a+b\left(x^{+}+x^{-}\right)+c x^{+} x^{-}}{\mathcal{J}\left(x^{+}-x^{-}\right)},
$$

where $a, b, c$ are arbitrary constants. It is easy to show that when the parameters, $a, b, c$ meet the following two conditions,

$$
\begin{aligned}
\mu & \equiv b^{2}-a c>0, \\
\phi_{B} & >\frac{\sqrt{\mu}}{\mathcal{J}},
\end{aligned}
$$

the solution describes a black hole. The steps detailing the calculation of mass are shown in appendix B.2 with the mass given by

$$
M=\frac{\mu}{16 \pi G \mathcal{J}}
$$

Doing an appropriate $\mathrm{SL}(2, R)$ transformation brings eq. (2.8) to the form eq. (A.2). Further coordinate transformations shown in appendix A can be done to bring the metric and the dilaton to the form eq. (A.7) and eq. (A.8) respectively. It then follows immediately that the temperature of the black hole is given by

$$
T=\frac{\sqrt{\mu}}{2 \pi}
$$

As discussed in $[8,9]$ the mass can be expressed in terms of the Schwarzian action. Let the proper time along the boundary be ${ }^{2} \hat{t}$, the boundary can be described by the function $t(\hat{t})$ and the mass is given by,

$$
M=-\frac{1}{8 \pi G \mathcal{J}} \operatorname{Sch}(t, \hat{t})
$$

\footnotetext{
${ }^{2}$ More correctly, in Fefferman-Graham coordinates the metric near the boundary takes the form, $\mathrm{d} s^{2}=$ $-\left(\frac{1+\mathcal{O}(\hat{z})^{2}}{\hat{z}^{2}}\right) \mathrm{d} \hat{t}^{2}+\frac{\mathrm{d} \hat{z}^{2}}{\hat{z}^{2}}$, and $\phi=\frac{1}{\mathcal{J} \hat{z}}$.
} 
where

$$
\operatorname{Sch}(t, \hat{t}) \equiv \frac{t^{\prime \prime \prime}(\hat{t})}{t^{\prime}(\hat{t})}-\frac{3}{2}\left(\frac{t^{\prime \prime}(\hat{t})}{t^{\prime}(\hat{t})}\right)^{2} .
$$

When the JT model arises from higher dimensional theories, $\phi$ is related to the area of the transverse sphere along the additional dimensions and its value at the horizon is proportional to the increase in area when the black hole is made non-extremal. This motivates the definition of the black hole entropy in the JT model to be

$$
S_{\mathrm{BH}}=\left.\frac{\phi}{4 G}\right|_{h} .
$$

The horizons lies along the loci where $(\nabla \phi)^{2}=0$. From eq. (2.8) it is easy to see that the future and past event horizons which correspond to the conditions $\partial_{-} \phi=0$ and $\partial_{+} \phi=0$ respectively lie at

and

$$
x_{h}^{+}=\frac{-b-\sqrt{\mu}}{c},
$$

$$
x_{h}^{-}=\frac{-b+\sqrt{\mu}}{c} .
$$

This leads to entropy, eq. (2.15),

$$
S_{\mathrm{BH}}=\frac{\sqrt{\mu}}{4 G \mathcal{J}} .
$$

From eq. (2.11) , eq. (2.12) and eq. (2.18) it is easy to see that the first law of thermodynamics

$$
T \mathrm{~d} S_{\mathrm{BH}}=\mathrm{d} M
$$

is obeyed by these black holes.

It is worth noting that our calculation for the mass leading to eq. (2.11) is carried out using the holographic renormalisation method and as explained in appendix B it is valid only for small temperatures,

$$
\frac{T}{\mathcal{J}} \ll \phi_{B}
$$

In this limit we are dealing with "small" black holes whose horizon radius is deep inside the boundary and the value of the dilaton at the event horizon $\phi_{h}$ meets the condition,

$$
\phi_{h} \ll \phi_{B} .
$$

However the agreement with the first law shows that the above expression for the mass eq. (2.11) should be valid for bigger black holes, at higher temperatures, as well.

\subsection{Infalling matter}

Let us next couple the JT model to classical matter. In the presence of matter the metric equations of motion become,

$$
-\frac{1}{8 \pi G}\left(\nabla_{\mu} \nabla_{\nu} \phi-g_{\mu \nu} \nabla^{2} \phi+g_{\mu \nu} \phi\right)=T_{\mu \nu}^{m},
$$

with $T_{\mu \nu}^{m}$ being the matter stress tensor. 
In the conformal gauge, (2.2), the components of eq. (2.22) become

$$
\begin{aligned}
-e^{2 \omega} \partial_{ \pm}\left(e^{-2 \omega} \partial_{ \pm} \phi\right) & =8 \pi G T_{ \pm \pm}^{m} \\
2 \partial_{+} \partial_{-} \phi+e^{2 \omega} \phi & =16 \pi G T_{+-}^{m} .
\end{aligned}
$$

We consider the special case of conformally invariant matter, with $T_{+-}^{m}=0$, which does not couple to the dilaton but only to the metric. Varying the dilaton in eq. (2.1), we get once again, eq. (2.3), which leads to the spacetime being $A d S_{2}$ as before. Let us also take the matter stress tensor to be purely infalling, i.e., $T_{++}^{m}=0$. In summary,

$$
T_{+-}^{m}=0=T_{++}^{m} .
$$

Also we take the $T_{--}^{m}$ component to satisfy

$$
T_{--}^{m}>0 \text {. }
$$

This is true if the matter satisfies the null energy condition (NEC)

$$
T_{a b}^{m} k^{a} k^{b}>0,
$$

with $k^{a}$ being the future-directed tangent vector for any null geodesic,

$$
k^{a}=\frac{\mathrm{d} x^{a}}{\mathrm{~d} \lambda},
$$

where $\lambda$ is an affine parameter along the geodesic. The ' ++ ' equation of (2.23) leads to,

$$
\phi=d\left(x^{-}\right)+\frac{h\left(x^{-}\right)}{x^{+}-x^{-}} .
$$

Then eq. (2.24) allows $d\left(x^{-}\right)$to be determined in terms of $h\left(x^{-}\right)$leading to

$$
\phi=\frac{1}{2} \partial_{-} h\left(x^{-}\right)+\frac{h\left(x^{-}\right)}{x^{+}-x^{-}} .
$$

The remaining ' -- ' component of (2.23) then gives,

$$
-\frac{1}{2} h^{\prime \prime \prime}=8 \pi G T_{--}^{m} .
$$

Let us note that eq. (2.31) follows from the boundary action

$$
I=-\frac{1}{8 \pi G \mathcal{J}} \int \mathrm{d} \hat{t} \operatorname{Sch}(t, \hat{t})+I^{m},
$$

where $\hat{t}$ is the boundary time and the boundary stress tensor $T_{\hat{t} \hat{t}}^{m}$ is obtained from the matter action $I^{m}$, when expressed as a functional of $t(\hat{t})$ on the boundary, as

$$
T_{\hat{t} \hat{t}}^{m}=t^{\prime} \frac{\delta I^{m}}{\delta t(\hat{t})} .
$$

This follows from eq. (B.23) as is discussed further in appendix B.2. 
Note that for $T_{--}^{m}=0$, eq. (2.31) has a general solution

$$
h_{0}=\frac{1}{\mathcal{J}}\left(a+2 b x^{-}+c\left(x^{-}\right)^{2}\right),
$$

for some constants $a, b, c$. It is easy to see that this, in conjunction with eq. (2.30), agrees with eq. (2.8) above.

Consider a situation where we start for $x^{-}<0$ with a solution of the form eq. (2.34) corresponding to a black hole and then allow matter to fall in. It follows from eq. (2.31) that the resulting solution is given by

$$
h=h_{0}-16 \pi G \int_{x^{-}=0} \int_{x^{-}=0} \int_{x^{-}=0} T_{--}^{m} .
$$

The initial black hole mass is given in eq. (2.11). The additional matter leads to a further increase in mass given by ${ }^{3}$

$$
\Delta M=\frac{\mathcal{J}}{2} \int \mathrm{d} x^{-} h T_{--}^{m} .
$$

The total mass is given in terms of $h$ (see appendix B.2) by

$$
M=\frac{\mathcal{J}}{64 \pi G}\left(h^{2}-2 h h^{\prime \prime}\right) .
$$

In the above expression, $h$ is evaluated at $z \rightarrow 0$ and prime indicates a derivative with respect to the Poincaré time $t$, see appendix $\mathrm{B}$ for more details. The calculation in appendix $\mathrm{B}$ is justified for black holes which are small, i.e. for which the value of the dilaton at the horizon meets the condition, eq. (2.21).

As is discussed in appendix B.2 in the more general time dependent case also, $M$ can be expressed in terms of the Schwarzian derivative as given in eq. (B.24). This is to be expected, since the Schwarzian term is the simplest one consistent with $\mathrm{SL}(2, R)$ symmetry.

From eq. (2.7) and eq. (2.30) we see that the boundary of spacetime can be expressed as a function of $x^{-}$,

$$
x^{+}=x^{-}+\frac{2 h\left(x^{-}\right)}{2 \phi_{B}-h^{\prime}\left(x^{-}\right)} .
$$

We see from eq. (2.35) and eq. (2.26) that $h$ becomes smaller as $x^{-}$increases. Generically $h$ cannot go to zero in finite proper time as measured on the boundary. This follows from the fact that boundary proper time goes as $\hat{t} \sim \int \frac{\mathrm{d} x^{-}}{h}$, and so near a first order zero, $x_{0}$, of $h$,

$$
\hat{t} \sim-\ln \left(x_{0}-x^{-}\right) .
$$

It is therefore enough to only consider the evolution till $h$ hits a zero, since matter cannot fall in thereafter from the boundary.

\footnotetext{
${ }^{3}$ Noting that $x^{ \pm}=t \pm z$ and using eq. (2.25), (B.23), we see that

$$
\Delta M=\frac{\mathcal{J}}{2} \int \mathrm{d} x^{-} h T_{--}^{m}=\frac{\mathcal{J}}{2} \int \mathrm{d} t h(t) T_{t t}^{m}=\int \mathrm{d} \hat{t} T_{\hat{t} \hat{t}}^{m} .
$$
}




\subsection{Second law}

Here we verify that for infalling matter of the type considered in the previous subsection, the second law is valid as long as the energy condition eq. (2.26) is satisfied. More specifically, we show below that the value of the dilaton increases monotonically along the event horizon. We had mentioned above that the horizon value of the dilaton plays the role of the horizon area and its monotonic increase is therefore the analogue of the area increase theorem in this system.

For simplicity we consider situations where the matter falls in for some duration (i.e. some interval in $x^{-}$) and then stops. The solution after the matter stops falling is then of the form, eq. (2.8), eq. (2.34), and has an event horizon (eh) located at eq. (2.16). We can choose an affine parameter $\lambda$ along the event horizon which meets the condition

$$
\frac{\mathrm{d} x^{-}}{\mathrm{d} \lambda}=\left(x_{h}^{+}-x^{-}\right)^{2} .
$$

It then follows that the first derivative,

$$
\frac{\mathrm{d} \phi}{\mathrm{d} \lambda}=\left.\left(x_{h}^{+}-x^{-}\right)^{2} \frac{\mathrm{d} \phi}{\mathrm{d} x^{-}}\right|_{\mathrm{eh}} .
$$

It is then easy to see that the second derivative satisfies the condition

$$
\frac{\mathrm{d}^{2} \phi}{\mathrm{d} \lambda^{2}}=\left.\left(x_{h}^{+}-x^{-}\right)^{4} \nabla_{-} \nabla_{-} \phi\right|_{\mathrm{eh}}=-8 \pi G\left(x_{h}^{+}-x^{-}\right)^{4} T_{--}^{m}<0
$$

where the second equality is due to the '--' equation of motion (2.23) and the last inequality follows from eq. (2.26).

Once the matter stops falling in, at late times,

$$
\left.\frac{\mathrm{d} \phi}{\mathrm{d} x^{-}}\right|_{\mathrm{eh}}=0
$$

since the event horizon is at $x^{+}=x_{h}^{+}$and the value of the dilaton at the event horizon is independent of $x^{-}$. From the inequality, eq. (2.43), it then follows that $\frac{\mathrm{d} \phi}{\mathrm{d} \lambda}>0$ along the event horizon, and thus $\phi$ monotonically increases.

\subsubsection{The apparent horizon}

It is interesting to note that in the classical system being analysed in this section the value of the dilaton at the apparent horizon $(a h)$ also increases monotonically. This will however turn out not to be necessarily true once we include the quantum effects of matter. At the apparent horizon,

$$
\left.\partial_{-} \phi\right|_{\mathrm{ah}}=0 .
$$

From eq. (2.30), this leads to the condition

$$
\left.x^{+}\right|_{\mathrm{ah}}=x^{-}-\frac{h^{\prime} \pm \sqrt{h^{\prime 2}-2 h h^{\prime \prime}}}{h^{\prime \prime}} .
$$


Requiring the value of the dilaton at the apparent horizon to be non-negative selects the upper sign for the discriminant, resulting in

$$
\begin{aligned}
\left.x^{+}\right|_{\mathrm{ah}} & =x^{-}-\frac{h^{\prime}+\sqrt{h^{\prime 2}-2 h h^{\prime \prime}}}{h^{\prime \prime}}, \\
\left.\phi\right|_{\mathrm{ah}} & =\frac{1}{2} \sqrt{h^{\prime 2}-2 h h^{\prime \prime}} .
\end{aligned}
$$

We can parametrise the trajectory of the apparent horizon by the coordinate $x^{-}$itself. We have,

$$
\left.\frac{\mathrm{d} \phi}{\mathrm{d} x^{-}}\right|_{\mathrm{ah}}=\left.\left(\frac{\partial \phi}{\partial x^{-}}+\frac{\partial \phi}{\partial x^{+}} \frac{\partial x^{+}}{\partial x^{-}}\right)\right|_{\mathrm{ah}}=\left.\left(\frac{-h}{\left(x^{+}-x^{-}\right)^{2}}\right) \frac{\partial x^{+}}{\partial x^{-}}\right|_{\mathrm{ah}},
$$

where we used eq. (2.45) and eq. (2.30). But we also have $\frac{\mathrm{d}\left(\partial_{-} \phi\right)}{\mathrm{d} x^{-}}=0$ along the apparent horizon. This gives

$$
\left.\partial_{-} x^{+}\right|_{\mathrm{ah}}=-\left.\frac{\partial_{-}^{2} \phi}{\partial_{+} \partial_{-} \phi}\right|_{\mathrm{ah}}=-\left.\frac{16 \pi G T_{--}^{m}}{e^{2 \omega} \phi}\right|_{\mathrm{ah}},
$$

where we used the equations of motion eqs. (2.23), (2.24), along with eq. (2.25) and eq. (2.45). Therefore using eq. (2.50) and the Poincaré metric eq. (2.4), eq. (2.49) becomes

$$
\left.\frac{\mathrm{d} \phi}{\mathrm{d} x^{-}}\right|_{\mathrm{ah}}=\left.4 \pi G \frac{h T_{--}^{m}}{\phi}\right|_{\mathrm{ah}} .
$$

Now we note that the r.h.s. in this equation is positive, since the energy condition, eq. (2.26) is met and $\phi$ and $h$ are positive. It therefore follows that the dilaton increases with increasing $x^{-}$, which is along the direction of increasing time. We also mention that while $h$ decreases with increasing $x^{-}$, eq. (2.35), it cannot turn negative in a finite duration of boundary time, see comments above after eq. (2.39). Finally, we also note from eq. (2.50) that in the presence of infalling matter the apparent horizon is space-like.

\section{Semi-classical analysis: the $\chi$ system}

We now turn to studying the effects of the quantum backreaction due to the matter fields. We will consider a simple system consisting of $N$ massless scalar fields which are minimally coupled with an the action (1.2). As was discussed in the introduction, we will work in the semiclassical limit obtained by taking $G \rightarrow 0$ and $N \rightarrow \infty$, keeping their product $G N$ fixed. In this limit, quantum effects of the matter fields need to be included while those of the gravity-dilaton fields can be neglected, since they are suppressed by $G$ and not enhanced by a factor of $N$. For later convenience, we find it useful to define the parameter

$$
\zeta=\frac{4 G N}{3} \text {. }
$$

Once quantum effects are included it is well known that the free scalar theory has a conformal anomaly. As a result, for the matter system above we get

$$
T_{\mu}^{\mu}=\frac{N}{24 \pi} R
$$

where $R$ is the Ricci scalar. 
The same value for $T_{\mu}^{\mu}$ can be obtained in a classical theory of a single scalar $\chi$ which is non-minimally coupled with an action:

$$
I_{\chi}=-\frac{N}{24 \pi}\left(\int \mathrm{d}^{2} x \sqrt{-g}\left(\partial_{\mu} \chi \partial^{\mu} \chi+\chi R\right)+2 \int_{\mathrm{bdy}} \sqrt{-\gamma} \chi K\right) .
$$

Note that the action has a prefactor which goes like $N$. It is easy to see that the resulting stress energy tensor gives $T_{\mu}^{\mu}$ in agreement with eq. (3.2).

One way to include the quantum effects of the $\psi_{i}$ fields is therefore to work with the classical $\chi$ theory, eq. (3.3), and couple it to the JT model. This system was analysed in [6] and we will study it first in this section. Following this in the next section, we will return to studying the system of the $\psi_{i}$ fields directly.

To completely specify the dynamics of this system we also need to specify the boundary condition satisfied by $\chi$. We will take $\chi$ to satisfy Dirichlet boundary conditions (specifically, $\left.\chi_{B}=0\right)$ at the boundary of spacetime where $\phi=\phi_{B}$.

\subsection{Thermodynamics}

To begin, we briefly review the thermodynamics of the JT model coupled to the matter theory with action eq. (3.3). This was analysed in detail in [6].

Since the $\chi$ field does not couple to the dilaton, varying the dilaton again gives eq. (2.3) and so the geometry still remains $A d S_{2}$. The black hole solution is conveniently described by writing the $A d S_{2}$ metric in Schwarzschild coordinates:

$$
\mathrm{d} s^{2}=-\left(r^{2}-\mu\right) \mathrm{d} t_{s}^{2}+\frac{\mathrm{d} r^{2}}{\left(r^{2}-\mu\right)} .
$$

The parameter $\mu$ determines the mass etc. of the black hole. The temperature is given by the expression (2.12) The metric eq. (3.4) is independent of $t_{s}$ and therefore a shift of the $t_{s}$ coordinate is manifestly a symmetry.

To proceed it is convenient to go to conformal coordinates,

$$
x_{s}^{ \pm} \equiv t_{s} \pm r_{*},
$$

where

$$
r_{*}=-\int \frac{\mathrm{d} r}{r^{2}-\mu}=-\frac{1}{2 \sqrt{\mu}} \ln \left(\frac{r-\sqrt{\mu}}{r+\sqrt{\mu}}\right) .
$$

In these coordinates the conformal factor, $\omega$, which appears in the metric eq. (2.2) is given by

$$
\omega=\frac{1}{2} \ln \left(r^{2}-\mu\right)
$$

The equation of motion for $\chi$, from the action eq. (3.3) is,

$$
\partial_{+} \partial_{-}(\chi+\omega)=0
$$

which gives,

$$
\chi=-\omega+f_{+}\left(x_{s}^{+}\right)+f_{-}\left(x_{s}^{-}\right)+c_{0},
$$


for some arbitrary functions $f_{+}$and $f_{-}$. Here $c_{0}$ is a constant. Requiring $\chi$ to be independent of $t_{s}$ fixes

$$
\begin{aligned}
& f_{+}\left(x_{s}^{+}\right)=p x_{s}^{+}, \\
& f_{-}\left(x_{s}^{-}\right)=-p x_{s}^{-},
\end{aligned}
$$

for some constant $p$. The components of the stress tensor for the $\chi$ field are,

$$
\begin{aligned}
& T_{+-}^{\chi}=\frac{N}{12 \pi} \partial_{+} \partial_{-} \chi \\
& T_{ \pm \pm}^{\chi}=\frac{N}{12 \pi}\left(-\partial_{ \pm}^{2} \chi+\partial_{ \pm} \chi \partial_{ \pm} \chi+2 \partial_{ \pm} \chi \partial_{ \pm} \omega\right) .
\end{aligned}
$$

Using the solution eq. (3.9), eq. (3.10) and eq. (3.11), the stress tensor components become

$$
\begin{aligned}
& T_{+-}^{\chi}=-\frac{N}{12 \pi} \partial_{+} \partial_{-} \omega \\
& T_{ \pm \pm}^{\chi}=\frac{N}{12 \pi}\left(\partial_{ \pm}^{2} \omega-\partial_{ \pm} \omega \partial_{ \pm} \omega\right)+\frac{N}{12 \pi} p^{2} .
\end{aligned}
$$

From the equations of motion for the ' +- ' component of the metric, eq. (2.24) with $T_{+-}^{m}$ replaced by eq. (3.14), we learn that

$$
\phi=\frac{r}{\mathcal{J}}+\frac{\zeta}{4}
$$

where we have used the definition (3.1). Here we have imposed that $\phi$ is independent of $t_{s}$ and regular at the horizon, ${ }^{4}$ which is given by

$$
r=\sqrt{\mu}
$$

$\mathcal{J}$ is the energy scale which breaks the scaling symmetry and is same as that appears in eq. (2.6). The ' ++ ' and ' -- ' components of the equations of motion (2.23) with $T_{ \pm \pm}^{m}$ replaced by $T_{ \pm \pm}^{\chi}$, eq. (3.15), then determines

$$
p=-\frac{\sqrt{\mu}}{2} .
$$

Note that with this value of $p$, the field $\chi$ is given by

$$
\chi=-\ln (r+\sqrt{\mu})+c_{0},
$$

and is non-singular at the horizon (3.17). Finally demanding that $\chi$ vanish at the boundary $\phi=\phi_{B}$ gives,

$$
c_{0}=\ln \left(\tilde{\phi}_{B} \mathcal{J}+\sqrt{\mu}\right) .
$$

Here $\tilde{\phi}_{B}$ is given by

$$
\tilde{\phi}_{B}=\phi_{B}-\frac{\zeta}{4} .
$$

\footnotetext{
${ }^{4}$ For $\phi$ which is independent of $t_{s}$ eq. (2.24) reduces to an ordinary second order equation in $r$ which has only one solution regular at the horizon.
} 
We will be working in the limit where eq. (2.20) is met. In this limit

$$
c_{0} \simeq \ln \left(\tilde{\phi}_{B} \mathcal{J}\right)
$$

so that

$$
\chi=-\ln (r+\sqrt{\mu})+\ln \left(\tilde{\phi}_{B} \mathcal{J}\right) .
$$

The ADM mass corresponding to the solution can be calculated as discussed in appendix $\mathrm{B}$ and is given by

$$
M=\frac{\mu}{16 \pi G \mathcal{J}}+\frac{N \sqrt{\mu}}{12 \pi} .
$$

The computation of the ADM mass shows that a counter-term is required to be added to the action eq. (3.3) to cancel divergences,

$$
I_{\mathrm{ct}}^{\chi}=-\frac{N}{24 \pi} \int_{\partial} \sqrt{-\gamma}
$$

We saw in the discussion of the previous section that the value of the dilaton at the horizon is analogous to the horizon area of higher dimensional gravity systems. Here we note that the $\phi$ and $\chi$ fields both couple to the curvature $R$ in the action, eq. (2.1) and (3.3). This motivated a definition of the generalized entropy [6]

$$
S_{\text {gen }}^{\chi}=\left.\frac{1}{4 G} \phi\right|_{h}-\left.\frac{N}{6} \chi\right|_{h}
$$

where $\left.\phi\right|_{h}$ and $\left.\chi\right|_{h}$ refer to the horizon values of the two fields.

Using eq. (3.16) and eq. (3.23), and noting that event horizon is given by eq. (3.17), we see that for the black hole solution under consideration,

$$
S_{\text {gen }}^{\chi}=\frac{1}{4 G}\left(\frac{\sqrt{\mu}}{\mathcal{J}}+\frac{\zeta}{2}\left(\ln \sqrt{\mu}+\frac{1}{2}-\ln \left(\frac{\tilde{\phi}_{B} \mathcal{J}}{2}\right)\right)\right) .
$$

It is easy to see from eq. (3.27), (3.24) and (2.12) that the system satisfies the first law of thermodynamics, $T \mathrm{~d} S_{\text {gen }}^{\chi}=\mathrm{d} M$. Moreover, using eq. (3.24), eq. (3.27) and eq. (2.12), we see that the temperature dependent part of the quantity $\beta F$, where $F=M-T S_{\text {gen }}^{\chi}$ is the free energy and $\beta=1 / T$, is given by

$$
\beta F=\beta M-S=-\frac{\sqrt{\mu}}{8 G \mathcal{J}}-\frac{\zeta}{8 G} \ln \sqrt{\mu} .
$$

Comparing this with the result obtained for the genus zero partition function obtained in [41], we find that the value of $\beta F$ in eq. (122) in [41] corresponds to $N=9$ in eq. (3.28), noting eq. (3.1).

Let us end with one comment. As mentioned in section 2.1, $\phi$ can be thought of as being analogous to the area of the horizon in this model. The extra contribution due to the $\chi$ field in $S_{\text {gen }}^{\chi}$, eq. (3.26), can be thought of as arising due to the entanglement of the $\psi_{i}$ matter fields across the horizon. The quantum effects of these fields have been replaced by the classical $\chi$ field here and their entanglement is replaced by the value of $\chi$ at the horizon. It is also worth noting that both $(\nabla \phi)^{2}$ and $\left(\nabla\left(\frac{\phi}{4 G}-\frac{N \chi}{6}\right)\right)^{2}$ vanish at the horizon. 


\section{$3.2 \quad$ Infalling matter}

Next we consider adding additional matter of the type considered in section 2.2. The matter is conformal and taken to be classical. We analyse how the behaviour of the system changes due to the additional effects of the $\chi$ field.

The matter does not couple to the dilaton and its stress tensor $T_{\mu \nu}^{m}$ satisfies the conditions, eq. (2.25) with $T_{--}^{m}$ being the only non-zero component.

The dilaton equation of motion shows that the metric continues to be $A d S_{2}$. The stress tensor for the $\chi$ field in the conformal gauge eq. (2.2) is given in eq. (3.12), (3.13). The equation of motion by varying $\chi$ is given in eq. (3.8) with the solution

$$
\chi=-\omega+f_{+}\left(x^{+}\right)+f_{-}\left(x^{-}\right)+\ln \left(2 \mathcal{J} \tilde{\phi}_{B}\right),
$$

where $\tilde{\phi}_{B}$ is defined in eq. (3.21), and the last term on r.h.s. is added to simplify the following discussion. Using eq. (3.29) in eqs. (3.12), (3.13) we get

$$
\begin{aligned}
& T_{+-}^{\chi}=-\frac{N}{12 \pi} \partial_{+} \partial_{-} \omega \\
& T_{ \pm \pm}^{\chi}=\frac{N}{12 \pi}\left(\partial_{ \pm}^{2} \omega-\partial_{ \pm} \omega \partial_{ \pm} \omega\right)+\frac{N}{12 \pi}\left(-\partial_{ \pm}^{2} f_{ \pm}+\left(\partial_{ \pm} f_{ \pm}\right)^{2}\right) .
\end{aligned}
$$

Here after, in this section, we work in Poincaré coordinates, eq. (2.4). In this case, $\partial_{ \pm}^{2} \omega-$ $\left(\partial_{ \pm} \omega\right)^{2}=0$, leading to

$$
T_{ \pm \pm}^{\chi}=\frac{N}{12 \pi}\left(-\partial_{ \pm}^{2} f_{ \pm}+\left(\partial_{ \pm} f_{ \pm}\right)^{2}\right) .
$$

For simplicity we consider situations where we start with empty $A d S_{2}$ into which the additional matter begins to fall at $x^{-}=0$. Before that, for $x^{-}<0$, the $\chi$ field is given by

$$
\chi=-\omega+\ln \left(2 \mathcal{J} \tilde{\phi}_{B}\right),
$$

with $f_{+}\left(x^{+}\right)=f_{-}\left(x^{-}\right)=0$ and satisfies the Dirichlet boundary condition. Also, the dilaton is given by,

$$
\phi=\frac{1}{2 \mathcal{J} z}+\frac{\zeta}{4} .
$$

It is easy to see that these solve the equations of motion. The term 'Poincaré vacuum' will be used to refer to the initial configuration eq. (3.33) and eq. (3.34) in the discussion related to $\chi$ system in the rest of the paper.

Once the additional matter begins to fall in, $T_{--}^{m}$ no longer vanishes. However since $f_{+}\left(x^{+}\right)$vanishes, $T_{++}^{\chi}=0$ and we learn from eq. (2.25) that

$$
T_{++}=T_{++}^{m}+T_{++}^{\chi}=0 .
$$

From the equations of motion for the ' +- ' (2.24), ' ++ ' eq. (2.23) components of the metric with $T_{\mu \nu}^{m}$ replaced by $T_{\mu \nu}^{\chi}+T_{\mu \nu}^{m}$ and using eq. (3.30), eq. (3.35), we learn that the dilaton is determined in terms of one function of $x^{-}$coordinate, $h\left(x^{-}\right)$by,

$$
\phi=\frac{h^{\prime}\left(x^{-}\right)}{2}+\frac{h\left(x^{-}\right)}{x^{+}-x^{-}}+\frac{\zeta}{4} \text {. }
$$


The value for $\phi$ given by eq. (3.34) corresponds to

$$
h=\frac{1}{\mathcal{J}} .
$$

The remaining equation involving $T_{--}$determines $h$ as

$$
h^{\prime \prime \prime}=-16 \pi G T_{--}^{\chi}-16 \pi G T_{--}^{m} .
$$

As was discussed above, we impose Dirichlet boundary condition for the field $\chi$ at the boundary $\phi=\phi_{B}$. From eq. (3.36), it follows that the boundary trajectory is given by

$$
z_{B}\left(x^{-}\right)=\frac{h}{2 \tilde{\phi}_{B}-h^{\prime}},
$$

where $\tilde{\phi}_{B}$ is given in eq. (3.21). As matter falls in, the form of $\chi$ is given by eq. (3.29) with $f_{+}=0$. By requiring that $\chi$ vanish at the boundary, we get

$$
f_{-}=-\ln \left(\frac{h}{2 \tilde{\phi}_{B}-h^{\prime}}\right)-\ln \left(2 \tilde{\phi}_{B} \mathcal{J}\right)
$$

leading to

$$
\chi=\ln z-\ln \left(\frac{h}{2 \tilde{\phi}_{B}-h^{\prime}}\right),
$$

where we used $\omega=-\ln z$ for the Poincaré metric, eq. (2.4). Therefore, from eq. (3.32), the $T_{--}^{\chi}$ component of the stress tensor becomes

$$
T_{--}^{\chi}=\frac{N}{12 \pi} \frac{\left(h h^{\prime \prime \prime}\left(2 \tilde{\phi}_{B}-h^{\prime}\right)+h^{\prime \prime}\left(2 h h^{\prime \prime}-h^{2}+4 \tilde{\phi}_{B}^{2}\right)\right)}{h\left(h^{\prime}-2 \tilde{\phi}_{B}\right)^{2}} .
$$

It is easy to see that eq. (3.38) now becomes a third order non-linear equation for $h$ in the presence of the source $T_{--}^{m}$ which is difficult to solve. To proceed we consider only situations where the infalling matter is varying slowly with $x^{-}$. More precisely, we take the frequency associated with this variation $\omega$, to satisfy the condition

$$
\frac{\omega}{\mathcal{J}} \ll \tilde{\phi}_{B}
$$

The reader will note that this is analogous to the condition imposed on the temperature $T$ in the previous section, eq. (2.20). For the kind of situations we consider, in this approximation, only the terms involving the minimum number of derivatives will survive; in the numerator of eq. (3.42) the surviving term goes like $4 \tilde{\phi}_{B}^{2} h^{\prime \prime}$, while in the denominator it goes like $4 \tilde{\phi}_{B}^{2} h$. Retaining these and neglecting the others gives,

$$
T_{--}^{\chi}=\frac{N}{12 \pi} \frac{h^{\prime \prime}}{h}
$$

leading to

$$
h^{\prime \prime \prime}=-\zeta \frac{h^{\prime \prime}}{h}-16 \pi G T_{--}^{m},
$$

where $\zeta$ is defined in eq. (3.1). This is a much simpler equation to solve. 
In the terms involving additional derivatives in eq. (3.42), each derivative is accompanied by an additional factor of $h$; we will self-consistently argue in appendix $\mathrm{C}$ that the condition eq. (3.43) is sufficient to suppress them.

We also note that when eq. (3.43) is met,

$$
\chi=\ln z-\ln \left(\frac{h}{2 \tilde{\phi}_{B}}\right) .
$$

The formula for the ADM mass, derived in B.3, is given by

$$
M=\frac{\mathcal{J}}{64 \pi G}\left(h^{\prime 2}-2 h h^{\prime \prime}-2 \zeta h^{\prime}\right) .
$$

From eq. (2.36) one finds that

$$
\partial_{\hat{t}} M=T_{\hat{t} \hat{t}}^{m}
$$

In the absence of infalling matter we see that $M$ is constant. Also for $T_{\hat{t} \hat{t}}^{m}>0, M$ increases.

\subsubsection{Some additional comments}

A few comments are in order here.

First, the reader might worry that the value of $\chi$ we start with at $x^{-}<0$, before matter begins to fall in, eq. (3.33), is in fact singular at the past Poincaré horizon. This follows from noting that

$$
\chi=-\omega=\ln z+\ln \left(2 \tilde{\phi}_{B} \mathcal{J}\right),
$$

and $z \rightarrow \infty$ at the horizon. It is best to regard this case as the limit of the finite temperature situation. For the the eternal black hole, in suitable Poincaré coordinates where $\phi$ is given by

$$
\phi=\frac{1-\mu x^{+} x^{-}}{\mathcal{J}\left(x^{+}-x^{-}\right)}
$$

$\chi$, eq. (3.19), takes the form

$$
\chi=\ln \left(\frac{x^{+}-x^{-}}{2}\right)-\ln \left(1+\sqrt{\mu} x^{+}\right)-\ln \left(1-\sqrt{\mu} x^{-}\right)+\ln \left(2\left(\tilde{\phi}_{B} \mathcal{J}+\sqrt{\mu}\right)\right) .
$$

This gives a finite value for $\chi$ at the horizon. In the limit $\mu \rightarrow 0$ we then get the value for $\chi$ in eq. (3.49).

Second, we can, in fact, start with a black hole of non-zero mass and redo the analysis of subsection 3.2 above. In this case, for $x^{-}<0, \chi$ and $\phi$ are given by eq. (3.51), eq. (3.50) respectively. In the subsequent evolution

$$
\chi=\ln z-\ln \left(1+\sqrt{\mu} x^{+}\right)+f_{-}\left(x^{-}\right),
$$

and $\phi$ is given by eq. (3.36) since eq. (3.35), (2.25) and eq. (3.30) continue to hold. It also follows from the Dirichlet boundary condition $\chi$ satisfies at the boundary that $f\left(x^{-}\right)$is

$$
f_{-}\left(x^{-}\right)=-\ln z_{B}\left(x^{-}\right)+\ln \left(1+\sqrt{\mu}\left(x^{-}+2 z_{B}\left(x^{-}\right)\right)\right),
$$


and therefore

$$
\chi=\ln z-\ln \left(1+\sqrt{\mu} x^{+}\right)-\ln z_{B}\left(x^{-}\right)+\ln \left(1+\sqrt{\mu}\left(x^{-}+2 z_{B}\left(x^{-}\right)\right)\right) .
$$

where $z_{B}\left(x^{-}\right)$is given by eq. (3.39). Then the '--' component of Einstein's equation, which determines $h$ by eq. (3.38), using eq. (3.53) and eq. (3.32), becomes

$$
\begin{aligned}
h^{\prime \prime \prime}= & -\zeta\left(\frac{\left(4 \tilde{\phi}_{B}^{2}-h^{\prime 2}+2 h h^{\prime \prime}\right)\left(2 \mu h+\left(1+\sqrt{\mu} x^{-}\right)\left(\left(1+\sqrt{\mu} x^{-}\right) h^{\prime \prime}-2 \sqrt{\mu} h^{\prime}\right)\right)}{h\left(2 \sqrt{\mu} h+\left(1+\sqrt{\mu} x^{-}\right)\left(2 \tilde{\phi}_{B}-h^{\prime}\right)\right)^{2}}\right) \\
& -\zeta\left(\frac{h h^{\prime \prime \prime}\left(1+\sqrt{\mu} x^{-}\right)\left(2 \sqrt{\mu} h+\left(1+\sqrt{\mu} x^{-}\right)\left(2 \tilde{\phi}_{B}-h^{\prime}\right)\right)}{h\left(2 \sqrt{\mu} h+\left(1+\sqrt{\mu} x^{-}\right)\left(2 \tilde{\phi}_{B}-h^{\prime}\right)\right)^{2}}\right)-16 \pi G T_{--}^{m} .
\end{aligned}
$$

We can simplify the above equation by using the approximation eq. (3.43) and also taking

$$
\frac{\sqrt{\mu} h}{\phi_{B}} \ll 1,
$$

which follows from eq. (2.20) if the initial temperature of the black hole we start with is small and $h \leq \frac{1}{\mathcal{J}}$ during the subsequent evolution. This gives, from eq. (3.55),

$$
h^{\prime \prime \prime}=-\zeta\left(\frac{h^{\prime \prime}}{h}-\frac{2 \sqrt{\mu}}{1+\sqrt{\mu} x^{-}} \frac{h^{\prime}}{h}+\frac{2 \mu}{\left(1+\sqrt{\mu} x^{-}\right)^{2}}\right)-16 \pi G T_{--}^{m} .
$$

It is straightforward to verify that

$$
h\left(x^{-}\right)=a_{1}\left(1+\sqrt{\mu} x^{-}\right)+a_{2}\left(1+\sqrt{\mu} x^{-}\right)^{2},
$$

is a solution to eq. (3.57), for arbitrary constants $a_{1}$ and $a_{2}$. We will show self-consistently in appendix C, that starting with a black hole which meets the condition, eq. (2.20) and with slowly infalling matter, which meets eq. (3.43), eq. (3.56) is also valid and neglecting the additional terms in eq. (3.55) leading to eq. (3.57) can be justified.

Finally, the mass formula, eq. (3.47) obtained above differs from the classical case, eq. (2.38). It was mentioned after eq. (2.38) that the expression in the classical system can be written in terms of the Schwarzian derivative and therefore preserves $\mathrm{SL}(2, R)$ invariance. It seems somewhat surprising at first therefore that an additional term is present in eq. (3.47), proportional to $\zeta h^{\prime}$. In fact this term is not proportional to the Schwarzian derivative and one might wonder how its presence is consistent with $\mathrm{SL}(2, R)$ invariance.

To understand this better we note that the starting forms for $\phi$ and $\chi$, eq. (3.50) and eq. (3.51) are not invariant under a general $\mathrm{SL}(2, R)$ transformation,

$$
x^{ \pm} \rightarrow \frac{p x^{ \pm}+q}{r x^{ \pm}+s}
$$

Under such a transformation, while $\phi$ continues to be given by the same form as eq. (3.36) with $h$ transforming as

$$
h\left(x^{-}\right) \rightarrow \frac{h\left(x^{-}\right)}{\left(r x^{-}+s\right)^{2}},
$$


$\chi$ assumes the form,

$$
\chi=\ln z-\ln \left(1+a x^{+}\right)+f_{-}\left(x^{-}\right),
$$

with

$$
a=\frac{\sqrt{\mu} s-r}{p-q \sqrt{\mu}} .
$$

More generally starting from $\chi$ as given in eq. (3.61), we would get $\chi$ of the same form with

$$
a \rightarrow \frac{a s-r}{p-q a} \text {. }
$$

One can then derive an expression for the mass by repeating the analysis above in the more general Poincaré coordinates obtained after doing such an $\mathrm{SL}(2, R)$ transformation, the details of which are shown in appendix B.3, to get

$$
M=\frac{\mathcal{J}}{64 \pi G}\left(h^{\prime 2}-2 h h^{\prime \prime}-2 \zeta\left(h^{\prime}-\frac{2 a h}{a t+1}\right)\right) .
$$

We see that there are two additional terms now compared to the classical expression. Using eq. (B.23) we can rewrite this expression in terms of the Poincaré time $t$ as, eq. (B.37)

$$
M_{\chi}=-\frac{1}{8 \pi G \mathcal{J}} \operatorname{Sch}(t, \hat{t})-\frac{N}{12 \pi}\left(\frac{t^{\prime \prime}}{t^{\prime}}-\frac{2 a t^{\prime}}{a t+1}\right),
$$

where $\hat{t}$ is the FG time coordinate and primes denote derivatives with respect to $\hat{t}$.

Under an $\operatorname{SL}(2, R)$ transformation it turns out that the two additional terms above are together also invariant, as discussed in appendix B.3. To summarise some of these comments, an additional parameter, $a$, enters in determining the mass for the $\chi$ system. It specifies the initial conditions for the $\chi$ field and enters in the second term on the r.h.s. in eq. $(3.61), \ln \left(1+a x^{+}\right)$. The presence of this additional parameter allows additional terms to arise in the mass formula, consistent with $\mathrm{SL}(2, R)$ invariance.

We had mentioned above that the equation of motion in the classical case arises from the Schwarzian action on the boundary coupled to the infalling matter stress tensor, eq. (2.32). We see from eq. (3.57) that due to the effects of the $\chi$ field there should be extra terms in the action; these also cannot arise from the Schwarzian derivative term, as in the case of the mass. We have not fully explored this issue but expect that due to the dependence on the extra parameter $a$ the resulting action giving rise to eq. (3.57) will also be $\operatorname{SL}(2, R)$ invariant.

\subsection{Detailed analysis: quasi normal mode}

Let us now return to the case considered in subsection 3.2 where we start with the Poincaré vacuum and $\chi$ is given in eq. (3.46) leading to the equation of motion, eq. (3.45). While this is a simpler equation to solve than the general case, since we have made the approximation, eq. (3.43), it is still quite non-trivial in the presence of general infalling matter.

Consider a situation where matter falls in for some time and then stops. One would expect that eventually the time dependence dies down and the system settles to a black hole state. This approach to equilibrium is determined by the quasi-normal modes of the 
system which characterise the final black hole and is independent of the details of the initial infalling matter. The quasi-normal modes describe the "ring down" of the black hole when subjected to external perturbations.

It turns out that in this system there is only one quasi-normal mode. Let us start with a black hole solution given by eq. (3.4), eq. (3.16), eq. (3.23) with mass $M$ given in eq. (3.24). A coordinate transformation eq. (C.25) now brings the metric in eq. (3.4) to the Poincaré form, with the dilaton taking the form eq. (3.36) with $h\left(x^{-}\right)$given by

$$
h=h_{0}=\frac{c_{1}}{\mathcal{J}}\left(x_{0}-x^{-}\right)
$$

where

$$
c_{1}=2 \sqrt{\mu},
$$

and

$$
x_{0}=\frac{1}{\sqrt{\mu}} .
$$

This coordinate transformation is being chosen with an eye to the discussion which follows. We note that in these $x^{+}, x^{-}$coordinates the future and past horizons, $\mathcal{H}^{ \pm}$, lie at $x^{+}=x_{0}$ and $x^{-}=-\infty$ respectively.

Let us denote the dilaton and $\chi$ perturbations in the quasi-normal mode as $\delta \phi, \delta \chi$ respectively. The quasi-normal mode should be regular at the future horizon, $x^{+}=x_{0}$.

In the Poincaré coordinates, the $\chi$ equation of motion leads to

$$
\delta \chi=\delta f_{+}\left(x^{+}\right)+\delta f_{-}\left(x^{-}\right) .
$$

Requiring that we start with the black hole solution and study its ring down leads to the condition that at $\mathcal{H}^{-}, \delta \chi$ vanish, leading to $\delta f_{+}=0$.

The ' ++ ' and ' + -' components of the metric equations then lead to the dilaton being of the form, eq. (3.36), with $h$ satisfying the source free equation, $\left(T_{--}^{m}=0\right)$ eq. (3.45),

$$
h^{\prime \prime \prime}=-\zeta \frac{h^{\prime \prime}}{h} .
$$

Expanding

$$
h=h_{0}+\delta h,
$$

we then get that the perturbation $\delta h$ satisfies the equation

$$
\delta h^{\prime \prime \prime}=-\zeta \frac{\delta h^{\prime \prime}}{h_{0}} .
$$

It is easy to see that the solution to this equation for a black hole of mass $M$ eq. (3.24) we started with is

$$
\delta h=-\frac{c_{2}}{\mathcal{J}(\alpha+2)}\left(x_{0}-x^{-}\right)^{\alpha+2}
$$

where

$$
\alpha=\frac{\zeta \mathcal{J}}{c_{1}} .
$$


and $c_{2}$ is an arbitrary constant. From eq. (3.36),(3.46) by considering sufficiently late times i.e., $x^{-}$close to $x_{0}$, and using the transformation to Schwarzschild coordinates, eq. (C.25) it then follows that

$$
\begin{aligned}
& \delta \phi \sim \frac{c_{2}}{2 \mathcal{J}} e^{-\left(\frac{\zeta \mathcal{J}}{2}+2 \pi T\right)\left(t_{s}-r_{*}\right)}, \\
& \delta \chi \sim \frac{c_{2}}{\zeta \mathcal{J}+8 \pi T} e^{-\left(\frac{\zeta \mathcal{J}}{2}+2 \pi T\right)\left(t_{s}-r_{*}\right)} .
\end{aligned}
$$

The universal exponent $\left(\frac{\zeta \mathcal{J}}{2}+2 \pi T\right)$ then characterizes the approach to the black hole solution. The quasi-normal mode is given by eq. (3.75) and eq. (3.76).

When $\zeta \rightarrow 0$ it is easy to see from eq. (3.73) that the solution for $\delta h$ corresponds to a black hole solution and therefore no quasi-normal mode exists. The mass corresponding to the perturbation eq. (3.73) can be computed by the linearized form of eq. (3.47) around eq. (3.66) and vanishes as expected.

Let us now consider a situation where starting with the Poincaré vacuum matter begins to fall in at $x^{-}=0$ and stops after some time. We take $T_{--}^{m}>0$ during the infall. At $x^{-}=0, h^{\prime}=h^{\prime \prime}=0$, since for $x^{-}<0, h$ is given by eq. (3.37) It follows from eq. (3.45) then that $h^{\prime \prime}$ must be negative for $x^{-}>0$, even after the matter stops falling in, and thus $h^{\prime}$ must also be negative for $x^{-}>0$ as discussed in appendix C. As a result $h$ will monotonically decrease and eventually vanish, say at $x^{-}=x_{0}$. Let us consider a case where $h>0$ when the matter stops falling in. In the subsequent evolution the mass is conserved and $h$ satisfies the source free equation, eq. (3.45), with $T_{--}^{m}=0$. Thus from eq. (3.66) and (3.73) we know that in the vicinity of $x_{0}$

$$
h=\frac{c_{1}}{\mathcal{J}}\left(x_{0}-x^{-}\right)-\frac{c_{2}}{\mathcal{J}(\alpha+2)}\left(x_{0}-x^{-}\right)^{\alpha+2} .
$$

and the system will "ring down" to the black hole corresponding to the final mass which determines $c_{1}$ in terms of mass $\mathrm{M}$ by eq. (3.67), eq. (3.24).

More details illustrating this behaviour are given in appendix $\mathrm{C}$.

We also note, as was discussed before in section 2.2 , that where $h \rightarrow 0$ in a generic way with a first order zero, boundary time $\hat{t} \rightarrow \infty$. Situations where this happens while matter is falling in, are less universal and need to be analysed on a case-to-case basis.

Let us end this subsection with some final comments. In the analysis above we took $\chi$ to be of the form, eq. (3.41) which is appropriate if we are starting with the Poincaré vacuum. Instead, if we started with a black hole of non-zero mass, the form $\chi$ takes is eq. (3.52). One can then repeat the quasi-normal mode analysis. In this case $h$ satisfies eq. (3.57), with the solution,

$$
h=\frac{1}{\mathcal{J}}\left(1-\mu\left(x^{-}\right)^{2}\right) .
$$

which is obtained for the choice $a_{1}=2$ and $a_{2}=-1$ in eq. (3.58). Denoting this solution as $h_{0}$ and considering a small fluctuation about it $\delta h$, eq. (3.71), we get that $\delta h$ satisfies the equation

$$
\delta h^{\prime \prime \prime}=-\zeta\left(\frac{2 \mu}{\left(1+\sqrt{\mu} x^{-}\right)^{2}} \frac{\delta h}{h_{0}}-\frac{2 \sqrt{\mu}}{1+\sqrt{\mu} x^{-}} \frac{\delta h^{\prime}}{h_{0}}+\frac{\delta h^{\prime \prime}}{h_{0}}\right) .
$$


General solution of the above equation for $\delta h$ can be written as a sum of three independent solutions as:

$$
\delta h=k_{1} \delta h_{1}+k_{2} \delta h_{2}+k_{3} \delta h_{3}
$$

where

$$
\begin{aligned}
& \delta h_{1}=1+\sqrt{\mu} x^{-} \\
& \delta h_{2}=\left(1+\sqrt{\mu} x^{-}\right)^{2}, \\
& \delta h_{3}=\left(1-\sqrt{\mu} x^{-}\right)^{\frac{\zeta \mathcal{J}}{2 \sqrt{\mu}}+2}\left(1+\sqrt{\mu} x^{-}\right)^{-\frac{\zeta \mathcal{J}}{2 \sqrt{\mu}}},
\end{aligned}
$$

and $k_{1}, k_{2}, k_{3}$ are arbitrary constants. Of these the first term can be shown to change the black hole mass, see eq. (3.58) and therefore cannot arise at late times. The second term, although does not alter the mass, can be set to zero by an $\mathrm{SL}(2, R)$ transformation. This leaves only the third solution eq. (3.83) which at late times, $x^{-} \rightarrow \frac{1}{\sqrt{\mu}}$, becomes

$$
\delta h_{3} \sim\left(1-\sqrt{\mu} x^{-}\right)^{2+\frac{\zeta \mathcal{J}}{2 \sqrt{\mu}}} .
$$

This is the quasi-normal mode with an exponent which agrees with what was obtained earlier, eq. (3.73), (3.74). In fact it is easy to see that for this solution, at late time, only the last term on the r.h.s. of eq. (3.79) contributes so that the perturbation satisfies eq. (3.72) obtained earlier.

This shows that the exponent $\left(\frac{\zeta \mathcal{J}}{2}+2 \pi T\right)$ which characterises the quasi-normal mode appears quite universally, regardless of the initial conditions for $\chi$, as would be expected on physical grounds.

\subsection{Second law}

In this subsection we show that the entropy $S_{\text {gen }}^{\chi}$ monotonically increases along the event horizon. The entropy is defined at the event horizon in eq. (3.26), and includes a contribution due to the $\chi$ field. We saw in section 3.1 that with this definition the First law is satisfied. Here we consider non-equilibrium situations with infalling matter of the kind that was considered in section 2.2. We will find that the second law is true as long as the energy condition, eq. (2.26) is satisfied.

We will restrict ourselves to situations where the matter stops falling in after some time. We saw that the solution is determined by the function $h$ which at late times $x^{-} \rightarrow x_{0}$, takes the form, eq. (3.77). The event horizon, where $(\nabla \phi)^{2}=0$, at late times, is given by

$$
x^{+}=x_{0} .
$$

From eq. (3.36) and using the form for $\chi$ eq. (3.41) we see that the entropy is,

$$
\begin{aligned}
S_{\text {gen }}^{\chi} & =\left.\left(\frac{\phi}{4 G}-\frac{N \chi}{6}\right)\right|_{\mathrm{eh}} \\
& =\left.\frac{1}{4 G}\left(\frac{h}{x_{0}-x^{-}}+\frac{h^{\prime}}{2}+\frac{\zeta}{4}-\frac{\zeta}{2}\left(\ln z-\ln \left(\frac{h}{2 \tilde{\phi}_{B}-h^{\prime}}\right)\right)\right)\right|_{\mathrm{eh}} .
\end{aligned}
$$


Note we have not assumed that the infalling matter is slowly varying with eq. (3.43) being met and our analysis below will apply to the general case.

To examine the second law, we proceed similar to the classical case discussed in section 2.3. The affine parameter along the event horizon is given by a relation analogous to eq. (2.41) given by

$$
\frac{\mathrm{d} x^{-}}{\mathrm{d} \lambda}=\left(x_{0}-x^{-}\right)^{2}=\left.4 e^{-2 \omega}\right|_{\mathrm{eh}}
$$

From eq. (3.86), we get

$$
\begin{aligned}
\frac{\mathrm{d} S_{\text {gen }}^{\chi}}{\mathrm{d} \lambda} & =\left(x_{0}-x^{-}\right)^{2} \nabla_{-} S_{\text {gen }}^{\chi} \\
& =\frac{\left(x_{0}-x^{-}\right)^{2}}{4 G}\left(\frac{h}{\left(x_{0}-x^{-}\right)^{2}}+\frac{h^{\prime}}{x_{0}-x^{-}}+\frac{h^{\prime \prime}}{2}+\frac{\zeta}{2}\left(\frac{1}{x_{0}-x^{-}}+\frac{h^{\prime}}{h}+\frac{h^{\prime \prime}}{2 \tilde{\phi}_{B}-h^{\prime}}\right)\right) .
\end{aligned}
$$

At late time, $x^{-} \rightarrow x_{0}, h$ is given by eq. (3.66) and we see that

$$
\frac{\mathrm{d} S_{\text {gen }}^{\chi}}{\mathrm{d} \lambda} \rightarrow 0
$$

Let us pause to make one comment here. We took $\chi$ as given in eq. (3.41) in obtaining eq. (3.86). More generally, starting with a black hole, instead of the Poincaré vacuum, $\chi$ is given by eq. (3.52), with $f_{-}\left(x^{-}\right)$in turn being expressed in terms of $h$ by eq. (3.53). In this case one can show that at late time $x^{-} \rightarrow x_{0}, h$ is given by a quadratic function,

$$
h=\tilde{k}_{1}\left(1+\sqrt{\mu} x^{-}\right)\left(x_{0}-x^{-}\right)
$$

for some constant $\tilde{k}_{1}$. Repeating the analysis above then shows that eq. (3.89) is valid in this more general situation as well.

Next we compute the second derivative of the entropy,

$$
\frac{\mathrm{d}^{2} S_{\text {gen }}^{\chi}}{\mathrm{d} \lambda^{2}}=\left.16 e^{-2 \omega} \partial_{-}\left(e^{-2 \omega} \partial_{-} S_{\text {gen }}^{\chi}\right)\right|_{\mathrm{eh}}
$$

From the '--' component of the Einstein equation, eq. (2.22) and noting that when the $\chi$ field is present, the stress tensor component $T_{--}$, including $T_{--}^{\chi}$ eq. (3.13), is given by,

$$
T_{--}=\frac{N}{12 \pi}\left(-\partial_{ \pm}^{2} \chi+\partial_{ \pm} \chi \partial_{ \pm} \chi+2 \partial_{ \pm} \chi \partial_{ \pm} \omega\right)+T_{--}^{m}
$$

we get

$$
e^{2 \omega} \partial_{-}\left(e^{-2 \omega} \partial_{-}\left(\phi-\frac{\zeta}{2} \chi\right)\right)=-\frac{\zeta}{2}\left(\partial_{-} \chi\right)^{2}-8 \pi G T_{--}^{m} .
$$

Using eq. (3.93) in eq. (3.91) we see that

$$
\frac{\mathrm{d}^{2} S_{\mathrm{gen}}^{\chi}}{\mathrm{d} \lambda^{2}}=\left.\frac{4 e^{-4 \omega}}{G}\left(-\frac{\zeta}{2}\left(\partial_{-} \chi\right)^{2}-8 \pi G T_{--}^{m}\right)\right|_{\mathrm{eh}}<0,
$$


where we have used the energy condition eq. (2.26). Since eq. (3.89) is met as $x^{-} \rightarrow x_{0}$, we conclude that

$$
\frac{\mathrm{d} S_{\mathrm{gen}}^{\chi}}{\mathrm{d} \lambda}>0
$$

showing that the generalised entropy monotonically increases along the future event horizon.

Let us note here that $x^{-} \rightarrow x_{0}$ corresponds to the "far future" since boundary proper time $\hat{t} \rightarrow \infty$ in this limit, eq. (2.40).

Before concluding this section let us note that unlike the classical case considered in section 2.3.1, in general the analogous statement for the apparent horizon, defined by eq. $(2.45)$, is not valid here, i.e. $S_{\text {gen }}^{\chi}$ now evaluated at the apparent horizon need not increase. We show this by considering an explicit example in appendix D. In contrast, when we consider the future Q-screen, which we will discuss in section 5, the generalised entropy does increase.

\section{Dynamical system with $\psi$ fields}

Having analysed the $\chi$ system in the previous section we now go back to the starting Lagrangian, eq. (1.2), and consider the system of $N$ scalars directly in the semi-classical limit, $N \rightarrow \infty, G \rightarrow 0$, keeping $G N$ fixed. We take the $\psi_{i}$ fields to satisfy Dirichtlet boundary condition

$$
\left.\psi_{i}\right|_{B}=0
$$

at the boundary, eq. (2.7), as was mentioned above. Let us consider a boundary described in conformal coordinates by the trajectory,

$$
x_{B}^{+}=x^{+}\left(x^{-}\right) .
$$

And consider the $x^{+}$modes to be in the vacuum with respect to a coordinate $x_{v}^{+}$. Then expanding the $\psi_{i}$ fields as

$$
\psi=\int_{0}^{\infty} d \omega\left[\frac{a_{+}(\omega)}{\sqrt{2 \omega}} e^{-i \omega x_{v}^{+}}+f\left(\omega, x^{-}\right)+\text {c.c. }\right]
$$

and imposing boundary conditions, eq. (4.1) gives

$$
f\left(\omega, x^{-}\right)=-\frac{a_{+}(\omega)}{\sqrt{2 \omega}} e^{-i \omega x_{v}^{+}\left(x_{B}^{+}\right)} .
$$

This shows that the $x^{-}$modes of $\psi_{i}$ fields will be in the vacuum with respect to a coordinate

$$
x_{v}^{-}=x_{v}^{+}\left(x_{B}^{+}\right) .
$$

We also note that the conformal anomaly is given by

$$
T_{+-}=-\frac{N}{12 \pi} \partial_{+} \partial_{-} \omega .
$$

Substituting in the ' +- ' component of the metric equation of motion, eq. (2.24) we get that

$$
2 \partial_{+} \partial_{-} \tilde{\phi}+e^{2 \omega} \tilde{\phi}=0
$$

where

$$
\tilde{\phi}=\phi-\frac{G N}{3} .
$$




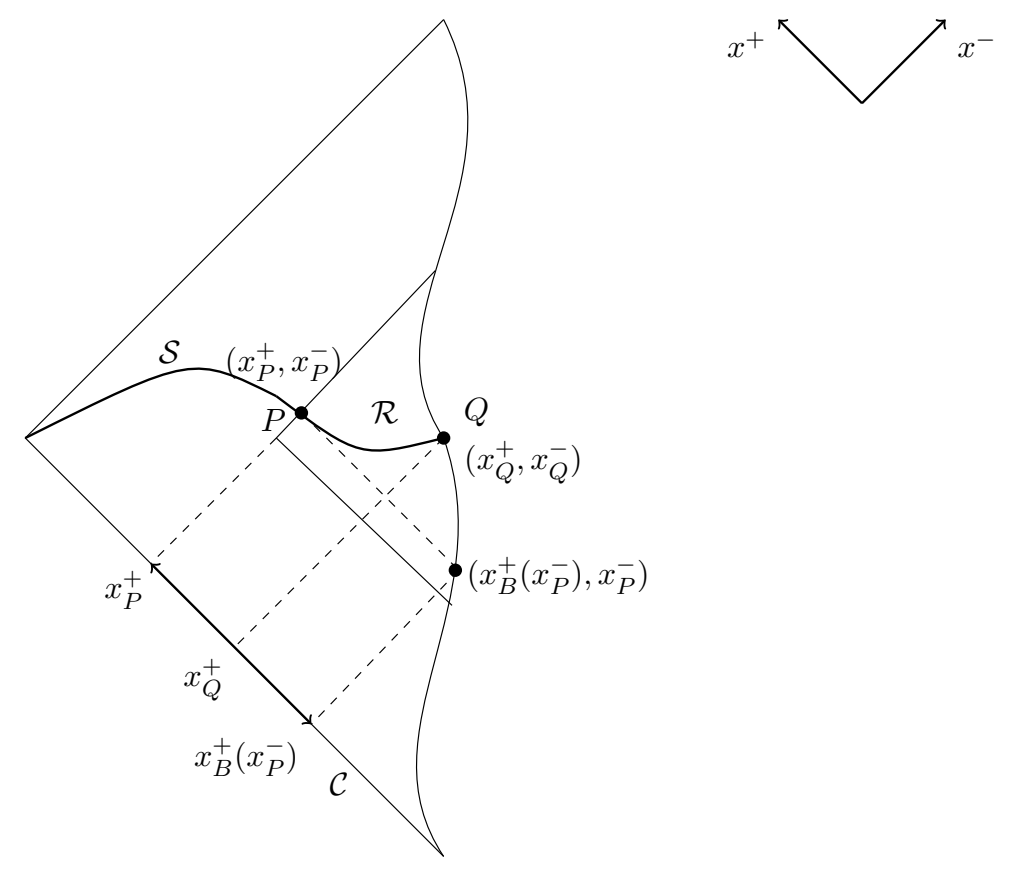

Figure 1. Entanglement of matter fields across the horizon.

\subsection{Entanglement entropy}

The entanglement entropy due to the scalar fields $\psi_{i}$ can be obtained using the results in $[138,139]$. Consider a single minimally coupled scalar $\psi$ and a space-like slice denoted as $\mathcal{S}$ in figure 1 which intersects the boundary at the point $Q$. We are interested in the entanglement entropy of the region extending from a point $P$ to $Q$ along $\mathcal{S}$. We will denote this region as $\mathcal{R}$ below. The entanglement entropy depends on the state of $\psi_{i}$. In the presence of the boundary the state of the left-moving modes ( $x^{-}$dependent) is determined by the state of the right-moving modes $\left(x^{+}\right.$dependent) and the location of the boundary, see eq. (4.5). Therefore it is enough to specify the state of the right-moving modes. We take this state to be in the vacuum with respect to the $x_{v}^{+}$coordinate. We will denote the coordinate of $P, Q$ as $x_{P}^{ \pm}, x_{Q}^{ \pm}$below. Now consider an initial null slice $\mathcal{C}$, the state of the left-movers can be specified on it. The right-moving modes present in region $\mathcal{R}$ of $\mathcal{S}$ correspond to right-movers on $\mathcal{C}$ that lie in the interval $\left[x_{P}^{+}, x_{Q}^{+}\right]$. The left-movers in $\mathcal{R}$ correspond to right-movers that lie in the interval $\left[x_{Q}^{+}, x_{B}^{+}\left(x_{P}^{-}\right)\right]$which after reflecting off the boundary have turned into left-movers. The entropy in $\mathcal{R}$ is therefore the entanglement in the right-moving modes lying in the region $\left[x_{P}^{+}, x_{Q}^{+}\right] \cup\left[x_{Q}^{+}, x_{B}^{+}\left(x_{P}^{-}\right)\right]$on $\mathcal{C}$.

It follows then from [138] that this entanglement entropy is given by

$$
S_{\mathrm{EE}}=\frac{1}{12}\left[\ln \frac{\left(\Delta x_{v}^{+}\right)^{2}}{\delta^{2}}+2 \rho_{P}\right] .
$$

Let us explain the different terms on the r.h.s. above. $\Delta x_{v}^{+}$is the length of the interval $\left[x_{P}^{+}, x_{Q}^{+}\right] \cup\left[x_{Q}^{+}, x_{B}^{+}\left(x_{P}^{-}\right)\right]$on $\mathcal{C}$ measured in the vacuum coordinate $x_{v}^{+}$. That is,

$$
\Delta x_{v}^{+}=x_{v}^{+}\left(x_{P}^{+}\right)-x_{v}^{+}\left(x_{B}^{+}\left(x_{P}^{-}\right)\right) .
$$


$\delta^{2}$ in eq. (4.9)) is an invariant cut-off which needs to be introduced to obtain a finite result. Finally, the metric in the coordinates $x_{v}^{ \pm}$is given by

$$
\mathrm{d} s^{2}=-e^{2 \rho} \mathrm{d} x_{v}^{+} \mathrm{d} x_{v}^{-},
$$

and $\rho_{P}$ is the conformal factor at the point $P$ in the $x_{v}^{ \pm}$coordinates

The result eq. (4.9) does not depend on the choice of the initial surface $\mathcal{C}$. Also, the vacuum state of the system is only specified up to an $\operatorname{SL}(2, R)$ transformation, since the notion of positive frequency modes does not change under such a transformation. It is easy to check that under such a transformation

$$
x_{v}^{+} \rightarrow \frac{a x_{v}^{+}+b}{c x_{v}^{+}+d}
$$

with $a d-b c=1, x_{v}^{-}$given by eq. (4.5) also transforms under the same $\mathrm{SL}(2, R)$ transformation

$$
x_{v}^{-} \rightarrow \frac{a x_{v}^{-}+b}{c x_{v}^{-}+d}
$$

and the entanglement entropy eq. (4.9) is invariant.

We will use eq. (4.9) in the discussion below often with the point $P$ lying on the horizon of black hole. Also, we will take all $N$ scalar fields to be in the same state. The full entanglement entropy will then be multiplied by a factor of $N$ and is given by

$$
S_{\mathrm{EE}}=\frac{N}{12}\left[\ln \frac{\left(\Delta x_{v}^{+}\right)^{2}}{\delta^{2}}+2 \rho_{h}\right]
$$

where $\rho_{h}$ denotes the conformal factor at the horizon.

\subsection{Thermodynamics}

We are now ready to study black holes in this system, our analysis parallels that of section 3.1 in the $\chi$ case. The black hole solution is given by the metric in eq. (3.4) and the dilaton given in eq. (3.16). We will show that this solution continues to solve the equations of motion in the $\psi$ system as well, for a suitable choice of vacuum for the matter fields. We will use both the conformal coordinates, $x_{s}^{ \pm}$given in eq. (3.5) and Kruskal coordinates, $X_{K}^{ \pm}$given in eq. (A.10) in the discussion below. We will also sometimes refer to the $x^{+}, x^{-}$ directions as "right-moving and left-moving " coordinates respectively

We take the $\psi_{i}$ fields to be in the vacuum with respect to $X_{K}^{ \pm}$coordinates. It then follows that the ' ++ ' and '--' components of the stress tensor in the $x_{s}^{ \pm}$coordinates are given by (see appendix A),

$$
\begin{aligned}
& T_{++}^{\psi}=-\frac{N}{24 \pi} \operatorname{Sch}\left(X_{K}^{+}, x_{s}^{+}\right), \\
& T_{--}^{\psi}=-\frac{N}{24 \pi} \operatorname{Sch}\left(X_{K}^{-}, x_{s}^{-}\right),
\end{aligned}
$$

and agree with the values they take in the $\chi$ system, eq. (3.15). This shows that the solution eq. (3.4) and eq. (3.16) satisfies the equations of motion. 
The temperature for the solution eq. (3.4) is given by eq. (2.12). The mass for the black hole in the $\psi$ system can be calculated as discussed in appendix B.4. In the limit eq. (2.20) the mass is given by

$$
M=\frac{1}{16 \pi G \mathcal{J}}\left(1-\frac{\zeta}{2 \tilde{\phi}_{B}}\right) \mu .
$$

Henceforth, we will work in the region of parameter space where

$$
1-\frac{\zeta}{2 \tilde{\phi}_{B}}>0
$$

We see from eq. (2.11), eq. (2.13) and (4.17) that the effect of the scalar fields can be incorporated by changing the coefficient of the Schwarzian action. One way to do this is to take

$$
G \rightarrow \frac{G}{1-\frac{\zeta}{2 \tilde{\phi}_{B}}},
$$

in eq. (2.11). In contrast in the $\chi$ system, the quantum effects result in a contribution to $M$ which goes like $\sqrt{\mu}$, eq. (3.24), and thus here the effects cannot be absorbed into a renormalisation of the coefficient of the Schwarzian action. Also, comparing the expressions for the mass in both the systems, eq. (4.17) and eq. (3.24), we see that the shift from the equilibrium mass is opposite in sign. ${ }^{5}$ It is unclear to us if a more general allowed boundary condition on the $\psi_{i}$ fields can reproduce the value for the mass as that obtained in the $\chi$ system.

Let us now calculate the generalised entropy at the horizon. This is given by

$$
S_{\mathrm{gen}}^{\psi}=S_{\mathrm{BH}}+S_{\mathrm{EE}}
$$

where $S_{\mathrm{BH}}$, defined in eq. (2.15), is the classical contribution and $S_{\mathrm{EE}}$ is the entanglement in the matter fields outside the horizon.

From eq. (3.16) and noting that the location of the horizon is given by eq. (3.17), we see that

$$
\phi_{h}=\frac{\sqrt{\mu}}{\mathcal{J}}+\frac{\zeta}{4}
$$

so that

$$
S_{\mathrm{BH}}=\frac{1}{4 G}\left(\frac{\sqrt{\mu}}{\mathcal{J}}+\frac{\zeta}{4}\right)
$$

In evaluating the entanglement entropy we need to take into account a subtlety. We took both the left-moving and right-moving modes to be in the Kruskal vacuum above. Let us, to begin, take the right-moving modes to be in the Kruskal vacuum, then since dilaton eq. (3.16) results in the boundary $\phi=\phi_{B}$ satisfying the equation,

$$
X_{k}^{+} X_{k}^{-}=-D
$$

\footnotetext{
${ }^{5}$ We acknowledge the referee for highlighting this.
} 
where

$$
D=\left(\frac{\tilde{\phi}_{B} \mathcal{J}-\sqrt{\mu}}{\tilde{\phi}_{B} \mathcal{J}+\sqrt{\mu}}\right),
$$

it follows from eq. (4.5) and eq. (4.23) that the left-moving modes would be in the vacuum with respect to the coordinate

$$
X_{v}^{-}=-\frac{D}{X_{k}^{-}}
$$

This is of course related to $X_{K}^{-}$by an $\operatorname{SL}(2, R)$ transformation and therefore $T_{--}^{\psi}$ will agree with eq. (4.16) above. However in computing the entanglement entropy one needs the value of the conformal factor $\rho_{h}$, eq. (4.14) in the coordinate system in which the matter fields are in the vacuum. For the choice we are making here $\rho_{h}$ must therefore be computed in the $\left(X_{K}^{+}, X_{v}^{-}\right)$coordinate system and not in the $\left(X_{K}^{+}, X_{K}^{-}\right)$coordinate system which would give a wrong result.

Once we keep this subtlety in mind the rest of the calculation is straightforward. It is convenient to calculate the entropy at the future (or past) horizon rather than at the bifurcate horizon. At the future horizon $X_{K}^{+}=0$, (see eq. (A.10)), one gets that $\rho_{h}$, eq. (4.11), using eq. (A.11) and noting eq. (4.25), is given by

$$
\rho_{h}=\left.\left(-\ln \frac{\left(1+X_{K}^{+} X_{K}^{-}\right)}{2}-\frac{1}{2} \ln \frac{D}{\left(X_{K}^{-}\right)^{2}}\right)\right|_{h}=-\frac{1}{2} \ln \frac{D}{4\left(X_{K}^{-}\right)^{2}} .
$$

From figure 1 we see that the interval $\Delta x_{v}^{+}$, eq. (4.10), is given by

$$
\Delta x_{v}^{+}=\left.\left(X_{K}^{+}-X_{v}^{-}\right)\right|_{h}=\frac{D}{X_{K}^{-}} .
$$

Thus the entanglement entropy for the $N$ fields, using eq. (4.14), becomes

$$
S_{\mathrm{EE}}=\frac{N}{12} \ln \left(\frac{4\left(\tilde{\phi}_{B} \mathcal{J}-\sqrt{\mu}\right)}{\tilde{\phi}_{B} \mathcal{J}+\sqrt{\mu}}\right)-\frac{N}{6 \pi} \ln (\delta) .
$$

The second term on the r.h.s. above is a constant independent of the temperature, ${ }^{6}$ it can be absorbed into a redefinition of the dilaton in $S_{\mathrm{BH}}$, when we evaluate the total entropy given in eq. (4.20). This is an example of the more general observation that UV divergences in the entanglement entropy can be absorbed in the renormalisation of $G_{N}$ [140-142], since the dilaton which is the coefficient of the Ricci scalar term in the action, eq. (2.1), plays the role of Newton's constant in this model.

In the approximation, eq. (2.20), the temperature dependent part of eq. (4.28) becomes,

$$
S_{\mathrm{EE}} \approx-\frac{N}{6} \frac{\sqrt{\mu}}{\tilde{\phi}_{B} \mathcal{J}} .
$$

As a result, from (4.20),eq. (4.22) and eq. (4.29) the generalised entropy is given by

$$
S_{\text {gen }}^{\psi}=\frac{1}{4 G \mathcal{J}}\left(1-\frac{\zeta}{2 \tilde{\phi}_{B}}\right) \sqrt{\mu} .
$$

\footnotetext{
${ }^{6}$ Reinstating $A d S_{2}$ length $L_{2}$, the $\delta$-dependent logarithmic term becomes $\ln \left(\frac{\delta}{L_{2}}\right)$.
} 
where we have dropped the terms that are independent of temperature. It is easy to see from eq. (4.30), (4.17) and eq. (2.12) that the first law of thermodynamics is met by the generalised entropy at the event horizon, i.e.,

$$
T \mathrm{~d} S_{\text {gen }}^{\psi}=\mathrm{d} M
$$

Let us end this discussion of the thermodynamics with some comments. First we see that in the $\psi$ system $S_{\text {gen }}^{\psi}$ is always positive,

$$
S_{\text {gen }}^{\psi}>0
$$

In contrast for the $\chi$ system it follows from eq. (3.27) and eq. (2.12) that the temperature dependent terms in the entropy are

$$
S_{\text {gen }}^{\chi}=\frac{\pi T}{2 G \mathcal{J}}+\frac{N}{6} \ln \left(\frac{T}{\mathcal{J} \tilde{\phi}_{B}}\right)
$$

and therefore at a sufficiently low $T, S_{\text {gen }}^{\chi}$ becomes negative probably indicating that the system no longer behaves in a sensible manner.

Second, in the discussion above in the $\psi$ system we started with the right-movers being in the Kruskal vacuum; instead if we had taken them to be in an $\operatorname{SL}(2, R)$ transformed vacuum and changed the left-movers' ground state also consistently under the same $\operatorname{SL}(2, R)$ transformation from the vacuum state we started with above, eq. (4.25), then the entanglement and hence generalised entropy would have remained unchanged from our result above, eq. (4.29). This follows from the comments towards the end of section 4.1. Finally, one can show that the metric eq. (3.4) and dilaton eq. (3.16) satisfy the equations of motion even when the approximation eq. (3.43) is not true, as long as the left and right-movers are in the Kruskal vacuum (or vacua related to them by an $\operatorname{SL}(2, R)$ transformation). ${ }^{7}$ This means that in general $S_{\text {gen }}^{\psi}$ is given by eq. (4.28). If we now assume the first law, we can calculate the mass of the system in this more general situation to be

$$
M=\frac{\mu}{16 \pi G \mathcal{J}}+\frac{N \tilde{\phi}_{B} \mathcal{J}}{24 \pi^{2}} \ln \left(\tilde{\phi}_{B}^{2} \mathcal{J}^{2}-\mu\right)
$$

\subsection{Infalling matter}

We now couple the system to an additional classical massless field and consider a situation where this massless field is purely infalling (left-moving) so that its stress tensor, which we denote as $T_{\mu \nu}^{m}$, has only one non-zero component, $T_{--}^{m}$. Instead of adding this extra field we could have considered a situation where there was an infalling coherent state made up of the $\psi_{i}$ matter fields themselves. This situation can also be analysed along the lines below, but has some additional complications which we would rather avoid here.

\footnotetext{
${ }^{7}$ This follows from the fact that eq. (3.16) can be written in the form eq. (3.36) in Poincaré coordinates, with $h=\frac{1}{\mathcal{J}}\left(1-\mu\left(x^{-}\right)^{2}\right)$, so that $h^{\prime \prime \prime}=0$; it then follows from eq. (4.37) that in Poincaré coordinates $T_{--}^{\psi}=0$.
} 
We choose the initial state for the $\psi_{i}$ fields to be the vacuum in the Poincare $x^{+}$ coordinate for the right-moving modes. The state for the left-moving modes is then given by eq. (4.5) to be the vacuum with respect to the coordinate

$$
x_{v}^{-}=x^{-}+\frac{2 h}{2 \tilde{\phi}_{B}-h^{\prime}} .
$$

Noting that in the Poincaré vacuum, $\left\langle x\left|T_{--}\right| x\right\rangle$, which is the first term on the r.h.s. of eq. (A.20), vanishes, we see that the stress tensor component $T_{--}^{\psi}$ in the vacuum state eq. (4.35) is given by

$$
T_{--}^{\psi}=-\frac{N}{24 \pi} \operatorname{Sch}\left(x_{v}^{-}, x^{-}\right)
$$

Using eq. (4.35) we get

$$
T_{--}^{\psi}=-\frac{N}{24 \pi}\left(\frac{-6 h^{2} h^{\prime \prime \prime 2}+2 h h^{\prime \prime \prime \prime}\left(2 h h^{\prime \prime}-h^{\prime 2}+4 \tilde{\phi}_{B}^{2}\right)+4 h^{\prime \prime \prime}\left(h^{\prime}+\tilde{\phi}_{B}\right)\left(2 h h^{\prime \prime}-h^{\prime 2}+4 \tilde{\phi}_{B}^{2}\right)}{\left(-2 h h^{\prime \prime}+h^{\prime 2}-4 \tilde{\phi}_{B}^{2}\right)^{2}}\right) .
$$

Again, we consider the slowly varying limit eq. (3.43). It this limit we get

$$
T_{--}^{\psi}=-\frac{N}{24 \pi} \frac{h^{\prime \prime \prime}}{\tilde{\phi}_{B}}
$$

It then follows that the equation for $h$ which is

$$
h^{\prime \prime \prime}=-16 \pi G T_{--}^{\psi}-16 \pi G T_{--}^{m},
$$

becomes

$$
\left(1-\frac{\zeta}{2 \tilde{\phi}_{B}}\right) h^{\prime \prime \prime}=-16 \pi G T_{--}^{m},
$$

where $\zeta$ is given by eq. (3.1).

We see in comparison with the classical case, eq. (2.31), that as in the discussion of the mass in eq. (4.17) above, the quantum effects of matter can be incorporated by changing the coefficient of the Schwarzian action, or equivalently by rescaling $G$ as given in eq. (4.19).

It then follows that $h$ is given in terms of $T_{--}^{m}$ by eq. (2.35) and the mass by eq. (2.38) after rescaling $G$ in subsection 2.2 by eq. (4.19). Starting with the Poincaré vacuum, consider the situation where matter starts falling in at $x^{-}=0$ and stops at $x^{-}=x_{f}$. Denoting

$$
\mu=\frac{8 \pi G \mathcal{J}}{\left(1-\frac{\zeta}{2 \tilde{\phi}_{B}}\right)} \int_{0}^{x_{f}} T_{--}^{m}\left(x^{-}\right) \mathrm{d} x^{-},
$$

we find that $h$, for $x^{-}>x_{f}$, is given by

$$
h\left(x^{-}\right)=\frac{1}{\mathcal{J}}-\frac{2}{\mathcal{J}} \int_{0}^{x^{-}}\left(\int_{0}^{x^{-}} \mu \mathrm{d} x^{-}\right) \mathrm{d} x^{-}=\frac{1}{\mathcal{J}}\left(1-\mu\left(x^{-}\right)^{2}\right)
$$


The expression for the dilaton with this $h\left(x^{-}\right)$is given by eq. (A.2). The mass can then be calculated using eq. (B.46) and is given by eq. (B.45).

Using the coordinate transformations mentioned in appendix $A$, we find that the metric, eq. (A.1) and the dilaton, eq. (A.2), transform to the form given by eq. (A.7) and eq. (A.8) respectively. It then follows that the temperature of the black hole corresponding to eq. (4.42) is given by the expression eq. (2.12), where $\mu$ is given by eq. (4.41). Also, the entropy will be given by the eq. (4.30). It then follows immediately that the system obeys the first law of thermodynamics, $T \mathrm{~d} S_{\text {gen }}^{\psi}=\mathrm{d} M$, which shows that the system equilibriates instantly after the matter ceases to fall in. It is easy to see from eq. (4.42), that $h$ vanishes at late times, $x^{-} \rightarrow \frac{1}{\sqrt{\mu}}$, as a linear zero, i.e.,

$$
h\left(x^{-}\right) \sim \frac{2}{\mathcal{J}}\left(1-\sqrt{\mu} x^{-}\right) .
$$

Let us end this subsection with some comments. In the $\chi$ system we found a quasinormal mode by studying small fluctuations around the black hole background. Here interestingly, it is easy to see from eq. (4.40) that once the matter stops falling in, the system instantaneously equilibriates to a black hole of the final mass. Thus, there is no non-trivial ringing down and therefore no quasi-normal mode is present.

If we had started with a black hole one would expect the $x^{+}$modes to be in the Kruskal vacuum instead of the Poincaré vacuum, which we considered as the initial state above. However, it is a curious fact that the Kruskal and Poincaré coordinates are related by an $\mathrm{SL}(2, R)$ transformation in this system, and therefore the two vacua are the same.

Finally, we had mentioned in the introduction that the differences in the $\chi$ and $\psi$ systems arise due to different boundary conditions. The $T_{+-}$component of the stress tensor agree in the two cases by construction. We also see from eq. (3.31), and eq. (3.61) that for the $\chi$ system, $T_{++}$vanishes, which is also true for the $\psi$ system if we start with the Poincaré or Kruskal vacua. Thus the differences in the two cases arise due to difference in $T_{--}$which in turn differs due to the different boundary conditions.

\subsection{Second law}

In this subsection we examine the second law for this system. To examine the second law, we proceed in a manner similar to section 3.4 and consider the first and second derivatives of the generalised entropy, $S_{\text {gen }}^{\psi}$, with respect to the affine parameter $\lambda$, eq. (2.41), along the event horizon. We again take the initial state for the $\psi_{i}$ fields to be in the vacuum in the Poincaré $x^{+}$coordinate for the right-moving modes. The vacuum for the left-moving modes is given by eq. (4.35). With this choice of vacuum state, and labelling the intersection point of the horizon and the spatial slice $\mathcal{S}$ as $\left(x_{0}, x^{-}\right)$, we find that $\rho_{h}$ in eq. (4.14) becomes

$$
\rho_{h}=\left.\left(\omega-\frac{1}{2} \ln x_{v}^{-\prime}\right)\right|_{h}
$$

where prime denotes a derivative with $x^{-}$and the horizon is located at $x^{+}=x_{0}$. Also, from fig 1 , the interval (4.10) is given by

$$
\Delta x_{v}^{+}=x_{0}-x_{v}^{-}\left(x^{-}\right) .
$$


Therefore, the entanglement entropy, (4.14), becomes

$$
S_{\mathrm{EE}}=\left.\frac{N}{6}\left(-\ln \left(\frac{x^{+}-x^{-}}{2}\right)-\frac{1}{2} \ln x_{v}^{-\prime}+\ln \left(x^{+}-x_{v}^{-}\right)\right)\right|_{h} .
$$

Consider the first derivative of $S_{\text {gen }}^{\psi}$ along the event horizon, $x^{+}=x_{0}$, given by

$$
\frac{\mathrm{d} S_{\mathrm{gen}}^{\psi}}{\mathrm{d} \lambda}=\left(x_{0}-x^{-}\right)^{2} \frac{\partial S_{\mathrm{gen}}^{\psi}}{\partial x^{-}}=\left(x_{0}-x^{-}\right)^{2}\left(\frac{\partial S_{\mathrm{BH}}}{\partial x^{-}}+\frac{\partial S_{\mathrm{EE}}}{\partial x^{-}}\right) .
$$

Using the forms of $S_{\mathrm{BH}}$, eq. (2.15), (3.36), and $S_{\mathrm{EE}}$, eq. (4.46), we get

$$
\frac{\mathrm{d} S_{\mathrm{gen}}^{\psi}}{\mathrm{d} \lambda}=\frac{\left(x_{0}-x^{-}\right)^{2}}{4 G}\left(\frac{h}{\left(x_{0}-x^{-}\right)^{2}}+\frac{h^{\prime}}{x_{0}-x^{-}}+\frac{h^{\prime \prime}}{2}+\frac{\zeta}{2}\left(-\frac{x_{v}^{-\prime \prime}}{2 x_{v}^{-\prime}}-\frac{x_{v}^{-\prime}}{x_{0}-x_{v}^{-}}+\frac{1}{x_{0}-x^{-}}\right)\right) .
$$

Using $x_{v}^{-}$given in eq. (4.35) and the late time form of $h$, eq. (4.43), we see that at late times,

$$
\frac{\mathrm{d} S_{\mathrm{gen}}^{\psi}}{\mathrm{d} \lambda} \rightarrow 0
$$

Consider the second derivative of the entropy along the horizon given by

$$
\frac{\mathrm{d}^{2} S_{\text {gen }}^{\psi}}{\mathrm{d} \lambda^{2}}=\left(x_{0}-x^{-}\right)^{2} \partial_{-}\left(\left(x_{0}-x^{-}\right)^{2} \partial_{-} S_{\text {gen }}^{\psi}\right) .
$$

Using eqs. (3.36) and (4.46), we get

$$
\begin{aligned}
\frac{d^{2} S_{\mathrm{gen}}^{\psi}}{\mathrm{d} \lambda^{2}}= & \frac{\left(x_{0}-x^{-}\right)^{4}}{8 G}\left(h^{\prime \prime \prime}+\frac{2 \zeta}{x_{0}-x^{-}}\left(\frac{x_{v}^{-\prime \prime}}{2 x_{v}^{-\prime}}+\frac{x_{v}^{-\prime}}{x_{0}-x_{v}^{-}}\right)\right) \\
& +\frac{\left(x_{0}-x^{-}\right)^{4}}{8 G} \zeta\left(\frac{1}{2}\left(\frac{x_{v}^{-\prime \prime}}{x_{v}^{-\prime}}\right)^{2}-\frac{x_{v}^{-\prime \prime \prime}}{2 x_{v}^{-\prime}}-\frac{\left(x_{v}^{-\prime}\right)^{2}}{\left(x_{0}-x_{v}^{-}\right)^{2}}-\frac{x_{v}^{-\prime \prime}}{x_{0}-x_{v}^{-}}-\frac{1}{\left(x_{0}-x^{-}\right)^{2}}\right) .
\end{aligned}
$$

We can simplify the above expression by using the equation of motion for $h$, eq. (4.39), which using $T_{--}^{\psi}$ in the form eq. (4.36), can be written as

$$
h^{\prime \prime \prime}=\frac{\zeta}{2}\left(\frac{x_{v}^{-\prime \prime \prime}}{x_{v}^{-\prime}}-\frac{3}{2}\left(\frac{x_{v}^{-\prime \prime}}{x_{v}^{-\prime}}\right)^{2}\right)-16 \pi G T_{--}^{m} .
$$

Therefore, using eq. (4.52), the expression eq. (4.51) simplifies to

$$
\frac{\mathrm{d}^{2} S_{\mathrm{gen}}^{\psi}}{\mathrm{d} \lambda^{2}}=-\frac{\left(x_{0}-x^{-}\right)^{4}}{8 G}\left(\frac{64 G^{2}}{\zeta}\left(\partial_{-} S_{\mathrm{EE}}\right)^{2}+16 \pi G T_{--}^{m}\right)
$$

which is manifestly negative if the energy condition eq. (2.26) is satisfied. From eq. (4.49) it then follows that $S_{\text {gen }}^{\psi}$ satisfies the condition

$$
\frac{\mathrm{d} S_{\text {gen }}^{\psi}}{\mathrm{d} \lambda}>0
$$

along the event horizon and asymptotically vanishes as $\lambda \rightarrow \infty$. This shows that the generalised entropy satisfies the second law along the future event horizon of the black hole. 


\section{$5 \quad$ Generalised entropy and Q-screens}

We saw in subsection 2.3.1 that in the classical theory the area of the apparent horizon also increases monotonically. More precisely, consider the co-dimension one surface which is foliated by marginally trapped surfaces (called the future Holographic screen in [131]). Then it was shown in subsection 2.3.1 that this surface is space-like and the area of the marginally trapped surface (i.e. the apparent horizon) increases monotonically as one goes outward, towards the boundary, along it. ${ }^{8}$ We also commented towards the end of subsection 3.4 that the area of the apparent horizon in the $\chi$ system did not grow in such a monotonic manner.

Here we consider the behaviour of the generalised entropy, instead of the area of the apparent horizon. A " future Q-screen" can be defined which is the analogue of the future holographic screen with the generalised entropy playing the role of the classical area. The idea is as follows, [132]. In the $2 \mathrm{D}$ spacetime we are considering here, we first define the quantum expansion to be the rate of change of the generalised entropy along a null ray. The marginally quantum trapped surface is then defined as a point on a Cauchy surface at which, a) the quantum expansion along the outward directed future null ray vanishes, and b) the quantum expansion along the inward directed future null ray is negative. ${ }^{9}$ With our choice of coordinates, this means

$$
\begin{aligned}
& \partial_{-} S_{\text {gen }}=0, \\
& \partial_{+} S_{\text {gen }}<0 .
\end{aligned}
$$

Finally, the future Q-screen is defined as a surface foliated by marginally quantum trapped surfaces.

We show below that the generalised entropy is a monotonic function along a future $\mathrm{Q}$ screen. This result follows in a very straightforward manner in 2D from another relation, called the Quantum Focussing Condition (QFC) which has also been discussed in the literature, [132].

In 2D the QFC takes the form,

$$
\frac{\mathrm{d}^{2} S_{\text {gen }}}{\mathrm{d} \lambda^{2}}<0
$$

where $\lambda$ is an affine parameter along a null geodesic. A related condition is called the Quantum Null Energy Condition (QNEC) which is given by

$$
\frac{\hbar}{2 \pi} \frac{\mathrm{d}^{2} S_{\mathrm{EE}}}{\mathrm{d} \lambda^{2}} \leq\left\langle T_{a b}\right\rangle k^{a} k^{b}
$$

with $\left\langle T_{a b}\right\rangle$ being the stress tensor which appears on the r.h.s. of Einstein's equations, and $k^{a}$ is the tangent vector along a null geodesic, eq. (2.28).

\footnotetext{
${ }^{8}$ As mentioned in subsection 2.1 the horizon value of the dilaton plays the role of the area in the JT model.

${ }^{9}$ In the $2 \mathrm{D}$ case being considered here the marginally quantum trapped surface is a point in spacetime.
} 
We will show below that eq. (5.3) is true in both the $\chi$ and $\psi$ systems, when the additional classical matter we add satisfies the NEC eq. (2.27).

We also show that the QNEC eq. (5.4) holds in both systems when the classical matter satisfies this condition. This will also allow us to establish that the generalised entropy is monotonic along a future Q-screen in both systems.

\subsection{The $\chi$ system}

We start with the $\chi$ system and first show that QFC is true. We take the definition of the generalised entropy in this case to be eq. (3.26)

Next, we take an outward directed null geodesic along increasing values of $x^{-}$. It then follows from eq. (3.26), and the discussion in subsection 3.4, see eq. (3.93) and eq. (3.94), that the QFC eq. (5.3) follows for matter satisfying the NEC, eq. (2.27). The analysis for a null geodesic along the $x^{+}$direction is entirely analogous, as long as the condition $T_{++}^{m} \geq 0$ is also met.

The role of the entanglement entropy in the $\chi$ system is played by

$$
S_{\mathrm{EE}} \rightarrow-\frac{N}{6} \chi
$$

Therefore QNEC eq. (5.4) takes the form (with $\hbar$ set equal to 1 ):

$$
\frac{-N}{12 \pi} \frac{\mathrm{d}^{2} \chi}{\mathrm{d} \lambda^{2}} \leq T_{a b} k^{a} k^{b}
$$

From eq. (3.26) it follows that QFC eq. (5.3) implies

$$
\frac{N}{12 \pi} \frac{\mathrm{d}^{2} \chi}{\mathrm{d} \lambda^{2}} \geq \frac{1}{8 \pi G} \frac{\mathrm{d}^{2} \phi}{\mathrm{d} \lambda^{2}} .
$$

From the equations of motion for $\phi$ it also follows that

$$
\frac{\mathrm{d}^{2} \phi}{\mathrm{d} \lambda^{2}}=-8 \pi G T_{a b} k^{a} k^{b} .
$$

Note here $T_{a b}$ is the full stress tensor including the contribution from the $\chi$ field. Combining eq. (5.7) and eq. (5.8) gives the QNEC condition eq. (5.6). We have set $\hbar=1$ above, but it is easily restored as follows. The prefactor $N$ in the $\chi$ action, eq. (3.3) arises from the conformal anomaly for the matter fields and should therefore actually be $N \hbar$, this gives exact agreement with the QNEC. ${ }^{10}$

We now turn to showing that $S_{\text {gen }}^{\chi}$ increases monotonically along a future Q-screen. From the QFC it follows that $\nabla_{-} \nabla_{-} S_{\text {gen }}^{\chi} \leq 0$. Using eq. (3.94) and equation of motion in the form eq. (3.93), it can be seen that along a future Q-screen, where eq. (5.1) is met,

$$
\partial_{-}^{2} S_{\mathrm{gen}}^{\chi}=-\frac{1}{4 G}\left(\frac{\zeta}{2}\left(\partial_{-} \chi\right)^{2}+8 \pi G T_{--}^{m}\right)<0 .
$$

\footnotetext{
${ }^{10}$ We also note that our normalisation for the stress tensor is correct; as a check this is the normalisation which arises after dimensional reduction from 4 dimensions.
} 
It then follows that no two points on a future Q-screen can have the same value of $x^{+}$. For if they did, the two points could be connected by a null ray along the $x^{-}$direction, and since eq. (5.9) is true, $\partial_{-} S_{\text {gen }}^{\chi}$ could not vanish at both points. We can therefore use the coordinate $x^{+}$to parametrise points on the future Q-screen. The monotonicity of $S_{\mathrm{gen}}^{\chi}$ along the Q-screen then follows simply by noting that eq. (5.2) is satisfied on it.

In fact we can say more about future Q-screens in the $\chi$ system.

For the system in initial Poincaré vacuum state and infalling null matter of the kind considered in subsection 3.2 the general solution is given in eq. (3.36), eq. (3.46). It then follows that

$$
\partial_{+} S_{\text {gen }}^{\chi}=-\frac{1}{4 G}\left(\frac{h}{\left(x^{+}-x^{-}\right)^{2}}+\frac{\zeta}{2\left(x^{+}-x^{-}\right)}\right)<0,
$$

(since $h>0$, as discussed in appendix C ).

This shows that $S_{\text {gen }}^{\chi}$ increases monotonically as one goes along the Q-screen towards the boundary.

In fact it can be easily seen that the future Q-screen in this case is a space-like surface. This follows by noting that the '+-' equation of motion eq. (2.24), where $T_{+-}^{m}$ is replaced by eq. (3.12), imply that

$$
\partial_{+} \partial_{-} S_{\text {gen }}^{\chi}=-\frac{1}{8 G} e^{2 \omega} \phi<0 .
$$

Since eq. (5.9) is also true it then follows from eq. (5.1) that, along the Q-screen,

$$
\frac{\mathrm{d} x^{-}}{\mathrm{d} x^{+}}=-\frac{\partial_{+} \partial_{-} S_{\text {gen }}^{\chi}}{\partial_{-}^{2} S_{\text {gen }}^{\chi}}<0,
$$

thereby showing that the future Q-screen is space-like.

\subsection{The $\psi$ system}

The generalised entropy which we denote by $S_{\text {gen }}^{\psi}$ in this case is defined in eq. (4.20). It follows from discussion in subsection 4.4, see eq. (4.53), that this generalised entropy also satisfies the condition

$$
\frac{\mathrm{d}^{2} S_{\mathrm{gen}}^{\psi}}{\mathrm{d} \lambda^{2}}<0
$$

for null geodesics along the $x^{-}$direction and classical matter satisfying the energy condition eq. (2.26). Similarly for null geodesics along the $x^{+}$direction, one can also show that eq. (5.13) is true as long as the condition $T_{++}^{m} \geq 0$ is met.

It also follows in a straightforward way from eq. (5.13) and the equations of motion for the dilaton that the QNEC is valid in this case. The reasoning is entirely analogous to steps eq. (5.6), (5.7), (5.8) in the $\chi$ system above.

We now turn to the behaviour of generalized entropy along a future Q-screen in this system. Since eq. (5.1) is met on a future Q-screen, it follows from eq. (5.13) that $\partial_{-}^{2} S_{\text {gen }}^{\psi}<$ 0 . It then follows, in a manner analogous to the discussion in the $\chi$ system that the Qscreen can be parametrised by the $x^{+}$coordinate and that $S_{\mathrm{gen}}^{\psi}$ is monotonically varying along the Q-screen.

It is worth mentioning that, in contrast with the $\chi$ system, the condition $\partial_{+} S_{\text {gen }}^{\psi}<0$ is not obviously met along a quantum marginal surface where $\partial_{-} S_{\text {gen }}^{\psi}=0$. 
We end with one final comment. One can also examine the existence of quantum extremal surfaces [143], where both conditions,

$$
\partial_{-} S_{\text {gen }}=\partial_{+} S_{\text {gen }}=0,
$$

are met. In the Kruskal extension of the Schwarzschild geometry (with two boundaries) one finds that there are additional spacetime points where eq. (5.14) is satisfied. However the value of $S_{\text {gen }}$ at these points is bigger than that at the bifurcate horizon. Thus the minimum value for $S_{\text {gen }}$ is obtained at the bifurcate horizon and equals the generalised entropy for the black hole, eq. (3.27), eq. (4.30), as one would expect on physical grounds.

\section{Conclusions}

In this paper we have considered the JT model coupled to matter in the semiclassical approximation. Two different models were analysed, in one case involving a matter field, $\chi$, with a non-minimal coupling, and in the other case $N$ scalar fields, $\psi_{i}, i=1, \cdots N$, which are massless and minimally coupled. While in both cases the matter has the same conformal anomaly, the boundary conditions which are imposed result in differences in the full matter stress tensor and the resulting behaviour of the two systems is also then different, see end of subsection 4.3.

In the $\psi$ case the effects of matter, in the semiclassical limit, is to renormalise the coefficient of the Schwarzian action as discussed in section $4 .{ }^{11}$ As a result the thermodynamics and response to additional infalling matter qualitatively stay the same as in the classical case. In contrast, for the $\chi$ system, analysed originally in [6], the effect of matter cannot be understood in this simple manner, and the thermodynamics and also dynamics change more appreciably.

Starting with the initial state, which corresponds to the Poincaré vacuum, when additional matter is thrown in, we find that in both cases a black hole forms, and the system thermalises. In the $\chi$ case, a quasi-normal mode characterises the ring-down to the final black hole geometry, once the matter stops falling in, while in the $\psi$ case the system instantly thermalises.

An interesting feature is that in both cases the second law of thermodynamics is obeyed in the following non-trivial way. One can define a generalised entropy which includes the area of the horizon, given in the JT theory by the horizon value of the dilaton, and a contribution due to the entanglement of matter. In the $\chi$ system this latter term is given by the horizon value of the $\chi$ field, while in the $\psi$ system it is the entanglement due to the $N$ free scalars across the black hole horizon. We show that this generalised entropy increases monotonically along the future event horizon of the black hole, if the extra infalling matter meets the null energy condition.

In earlier work, [134], a future Q-screen was defined which is the analogue of the locus of an apparent horizon with the generalised entropy now playing the role of the horizon

\footnotetext{
${ }^{11}$ More correctly this was shown to be true at small temperature $\frac{T}{\mathcal{J}} \ll \phi_{B}$, and small frequency, $\frac{\omega}{\mathcal{J}} \ll \phi_{B}$ in section 4 .
} 
area. Interestingly, we find that in both systems the Quantum Focussing Condition is satisfied, and as a result the generalised entropy also increases along a future Q-screen.

We have not been able to analyse general time dependent situation here and have restricted ourselves to cases where eq. (3.43) is met i.e., the additional matter falls in slowly. It would be interesting to study more general situations as well.

We have worked here in the semi-classical limit where the 2D Newton constant, $G \rightarrow 0$, and $N \rightarrow \infty$, keeping $G N$ fixed. Going forward it would be interesting to also incorporate the quantum effects of the gravity-dilaton sector systematically, order by order in $G$, and to compare the results with those obtained in [41, 80].

In this context it is worth keeping the following observations in mind. The JT model can be obtained by dimensional reduction from higher dimensions, as discussed in [18, 30, 48]. The near horizon $A d S_{2}$ geometry obtained in this way is then glued in to the higher dimensional asymptotically flat or AdS space. It would be worth investigating how this gluing of the geometry to the higher dimensional asymptotic spacetime affects the results for the higher loop corrections to the partition function etc. Also, on carrying out a dimensional reduction extra terms arise in the action; in particular a term quadratic in the dilaton of the form, [18],

$$
\Delta S=C_{1} \int \mathrm{d}^{2} x \sqrt{-g} \phi^{2} .
$$

Due to these extra terms, the geometry in the near-horizon region is no longer $A d S_{2}$ and departs from it, at the same order as $\phi$, in the parameter $1 / \mathcal{J}$, eq. (2.6). It was already noted in earlier work that these departures can have significant effects in the semi-classical limit discussed here [144]. Incorporating them while studying the loop corrections in $G$ could also be important.

It is worth digressing briefly to remind the reader why the corrections to the action which arise can lead to significant effects in the semi-classical limit. In general the 2D metric for a static black hole can be written as

$$
d s^{2}=f(r)\left(-d t_{s}^{2}+d r_{*}^{2}\right)
$$

where

$$
r_{*}=-\int \frac{d r}{f(r)}
$$

At extremality, $f(r)$ has a second order zero at the horizon $r=r_{h}$. If one calculates the quantum back reaction due to a massless scalar field which is in the vacuum with respect to $x_{s}^{ \pm}=t_{s} \pm r_{*}$ one finds that, at the future event horizon, this is given by

$$
T_{\mu \nu} U^{\mu} U^{\nu} \sim \frac{f^{\prime \prime \prime}}{f^{\prime}},
$$

where $U^{\mu}$ is the 4 -velocity of a freely infalling observer. In the JT model where the geometry is exactly $A d S_{2}, f(r)=\left(\frac{r-r_{h}}{r_{h}}\right)^{2}$ and thus $f^{\prime \prime \prime}$ vanishes, so that the r.h.s. vanishes. However, more generally once, say, the effects of the $\phi^{2}$ term are incorporated, the metric will not be $A d S_{2}$ and in general $f^{\prime \prime \prime}$ will not vanish as one approaches the horizon. For example for the $4 \mathrm{D}$ extremal $\mathrm{RN}$ case we have

$$
f(r)=\frac{\left(r-r_{h}\right)^{2}}{r^{2}},
$$


and $f^{\prime \prime \prime}\left(r_{h}\right)$ does not vanish. It then follows from eq. (6.4) that since $f^{\prime}$ will continue to vanish at the horizon, due to the second order zero at extremality, the r.h.s. of eq. (6.4) will diverge. This means a freely infalling observer will see a diverging energy density at the future horizon. This signals the possibility that the back reaction due to quantum effects will not be small. In fact it was shown in [144] that the quantum effects do significantly change the behaviour of the geometry near the horizon in the case of the $2 \mathrm{D}$ system obtained after dimensionally reducing the $\mathrm{RN}$ black hole from $4 \mathrm{D}$.

Returning to the main thread of our discussion it would clearly then be worth going beyond the semiclassical limit studied here and incorporating the quantum corrections order by order in $G$, keeping some of the observations above also in mind.

In conclusion, it is remarkable that the JT model, which is a simple model of gravity, is proving to be such a rich laboratory for studying various aspects of quantum gravity. Recent developments [41, 50, 145-147], suggest that it can provide interesting insights both to the information puzzle and towards understanding the Euclidean path integral of quantum gravity. In addition, it could be an important laboratory for understanding the different types of bulk entropy associated with different types of horizons and surfaces. We look forward to these developments with considerable anticipation.

\section{Acknowledgments}

We would like to thank Ahmed Almheiri, Matthew Headrick, Gautam Mandal, Juan Maldacena, Shiraz Minwalla, Aron Wall for valuable discussions. We thank the DAE, Government of India, for support. SPT also acknowledges support from the J. C. Bose fellowship of the DST, Government of India and the organisers of the workshop on Quantum Information and String Theory 2019, YITP, June 2019. UM thanks the organisers of The 36th Advanced School in Physics on Recent Progress in Quantum Field/String Theory at the Israel Institute for Advanced Studies, The Hebrew University of Jerusalem, Israel, the Spring School on Superstring Theory and Related Topics at the Abdus Salam International Centre for Theoretical Physics, Italy, and the It from Qubit School / Workshop (Quantum Information and String Theory 2019) at the Yukawa Institute for Theoretical Physics, Kyoto University, Japan for their warm hospitality while this work was in progress. VV would like to thank the organisers of the Spring School on Superstring Theory and related topics at ICTP, Trieste, for their hospitality during the course of this work. We gratefully acknowledge support from the Infosys Endowment for Research on the Quantum Structure of Spacetime. Most of all, we thank the people of India for generously supporting research in String Theory.

\section{A Coordinate transformations}

In this appendix, for completeness, we give details of the transformations between the Poincaré, Schwarzschild and Kruskal coordinate systems and also discuss the transformation properties of the matter stress tensor. 
The Poincaré metric in light cone coordinates is given by (2.4),

$$
\mathrm{d} s^{2}=-\frac{4 \mathrm{~d} x^{+} \mathrm{d} x^{-}}{\left(x^{+}-x^{-}\right)^{2}} .
$$

Consider the vacuum dilaton solution given in eq. (2.8), with the condition eq. (2.9) being met. We can do an appropriate $\mathrm{SL}(2, R)$ transformation to get the dilaton solution eq. (2.8) to the form

$$
\phi=\frac{1}{\mathcal{J}}\left(\frac{1-\mu x^{-} x^{+}}{x^{+}-x^{-}}\right)
$$

The relation between these Poincaré coordinates and Schwarzschild coordinates is given by,

$$
x^{ \pm}=\frac{1}{\sqrt{\mu}} \tanh \left(\frac{\sqrt{\mu} x_{s}^{ \pm}}{2}\right)
$$

under which the Poincaré metric eq. (A.1) becomes

$$
\mathrm{d} s^{2}=-\frac{\mu \mathrm{d} x_{s}^{+} \mathrm{d} x_{s}^{-}}{\sinh ^{2}\left(\frac{\sqrt{\mu}}{2}\left(x_{s}^{+}-x_{s}^{-}\right)\right)},
$$

and the dilaton, eq. (A.2), transforms to

$$
\phi=\frac{\sqrt{\mu}}{\mathcal{J}} \operatorname{coth}\left(\frac{\sqrt{\mu}}{2}\left(x_{s}^{+}-x_{s}^{-}\right)\right) .
$$

We can convert the metric eq. (A.4) into static coordinates by writing it in terms of the coordinates $t_{s}$ and $r_{*}$, where $x_{s}^{ \pm}=t_{s} \pm r_{*}$. Doing so gives

$$
\mathrm{d} s^{2}=\frac{\mu}{\sinh ^{2} \sqrt{\mu} r_{*}}\left(-\mathrm{d} t_{s}^{2}+\mathrm{d} r_{*}^{2}\right)
$$

Using the relation eq. (3.6) between $r$ and $r_{*}$, the metric and the dilaton expressed in the coordinates $t_{s}, r$ become

$$
\begin{aligned}
\mathrm{d} s^{2} & =-\left(r^{2}-\mu\right) \mathrm{d} t_{s}^{2}+\frac{\mathrm{d} r^{2}}{r^{2}-\mu}, \\
\phi & =\frac{r}{\mathcal{J}} .
\end{aligned}
$$

We define the Kruskal coordinates $X_{K}^{+}, X_{K}^{-}$to be

$$
X_{K}^{-}=e^{\sqrt{\mu} x_{s}^{-}}, \quad X_{K}^{+}=-e^{-\sqrt{\mu} x_{s}^{+}} .
$$

The Poincaré coordinates $x^{ \pm}$and the Kruskal coordinates $X_{K}^{ \pm}$are related as

$$
\begin{aligned}
& X_{K}^{+}=\left(\frac{\sqrt{\mu} x^{+}-1}{\sqrt{\mu} x^{+}+1}\right), \\
& X_{K}^{-}=\left(\frac{1+\sqrt{\mu} x^{-}}{1-\sqrt{\mu} x^{-}}\right) .
\end{aligned}
$$


Using the transformation eq. (A.10), the Poincaré metric eq. (A.1) becomes

$$
\mathrm{d} s^{2}=-\frac{4 \mathrm{~d} X_{K}^{+} \mathrm{d} X_{K}^{-}}{\left(1+X_{K}^{+} X_{K}^{-}\right)^{2}} .
$$

Next let us discuss how the stress tensor of a massless free scalar field transforms under a simultaneous change of coordinates and the vacuum. We consider two coordinate systems denoted by $\left(X^{+}, X^{-}\right)$and $\left(x^{+}, x^{-}\right)$with metric,

$$
\begin{aligned}
\mathrm{d} s^{2} & =-F\left(X^{+}, X^{-}\right) \mathrm{d} X^{+} \mathrm{d} X^{-}, \\
\mathrm{d} s^{2} & =-f\left(x^{+}, x^{-}\right) \mathrm{d} x^{+} \mathrm{d} x^{-} .
\end{aligned}
$$

The stress tensor in the $X^{ \pm}$coordinates when the system is in the $X^{ \pm}$vacuum, i.e. annihilated by positive frequency modes of $X^{ \pm}$, is given by [148-150]

$$
\begin{aligned}
\left\langle X\left|T_{\mu \nu}\right| X\right\rangle & =\Theta_{\mu \nu}+\frac{1}{48 \pi} R g_{\mu \nu} \\
\Theta_{ \pm \pm} & =-\frac{1}{12 \pi} F^{\frac{1}{2}} \partial_{X^{ \pm}}^{2} F^{-\frac{1}{2}}, \quad \Theta_{+-}=0 .
\end{aligned}
$$

Using eq. (A.15), let us compute the value of the stress tensor in the coordinates $x^{ \pm}$. Comparing eq. (A.12) and eq. (A.13), we see that

$$
f=F \frac{\mathrm{d} X^{+}}{\mathrm{d} x^{+}} \frac{\mathrm{d} X^{-}}{\mathrm{d} x^{-}} .
$$

The stress tensor transforms as a rank 2 tensor and so we have

$$
\begin{aligned}
\left\langle X\left|T_{x^{+} x^{+}}\right| X\right\rangle & =-\frac{1}{12 \pi}\left(\frac{\mathrm{d} X^{+}}{\mathrm{d} x^{+}}\right)^{2} F^{\frac{1}{2}} \partial_{X^{ \pm}}^{2} F^{-\frac{1}{2}} \\
& =-\frac{1}{12 \pi}\left(\frac{\mathrm{d} X^{+}}{\mathrm{d} x^{+}}\right)^{2}\left(\frac{f}{\frac{\mathrm{d} X^{+}}{\mathrm{d} x^{+}} \frac{\mathrm{d} X^{-}}{\mathrm{d} x^{-}}}\right)^{\frac{1}{2}} \frac{\mathrm{d} x^{+}}{\mathrm{d} X^{+}} \frac{\partial}{\partial x^{+}}\left(\frac{\mathrm{d} x^{+}}{\mathrm{d} X^{+}} \frac{\partial}{\partial x^{+}}\left(\frac{f}{\frac{\mathrm{d} X^{+}}{\mathrm{d} x^{+}} \frac{\mathrm{d} X^{-}}{\mathrm{d} x^{-}}}\right)^{-\frac{1}{2}}\right) .
\end{aligned}
$$

Simplifying, we get

$$
\begin{aligned}
\left\langle X\left|T_{x^{+} x^{+}}\right| X\right\rangle & =-\frac{1}{12 \pi} f^{\frac{1}{2}} \partial_{x^{+}}^{2} f^{-\frac{1}{2}}-\frac{1}{24 \pi}\left(\frac{\left(X^{+}\right)^{\prime \prime \prime}}{\left(X^{+}\right)^{\prime}}-\frac{3}{2}\left(\frac{\left(X^{+}\right)^{\prime \prime}}{\left(X^{+}\right)^{\prime}}\right)^{2}\right) \\
& =-\frac{1}{12 \pi} f^{\frac{1}{2}} \partial_{x^{+}}^{2} f^{-\frac{1}{2}}-\frac{1}{24 \pi} \operatorname{Sch}\left(X^{+}, x^{+}\right),
\end{aligned}
$$

where $\left(X^{+}\right)^{\prime}=\frac{\mathrm{d} X^{+}}{\mathrm{d} x^{+}}$. An analogous relation exists for ' -- ' components by replacing all ' + ' indices with ' - ' indices. The first term in the final expression, using eq. (A.14),eq. (A.15), can be interpreted as value of the stress tensor in the coordinates $x^{ \pm}$with the system also being in the vacuum with respect to the $x^{ \pm}$coordinates, that is

$$
\left\langle x\left|T_{x^{+} x^{+}}\right| x\right\rangle=-\frac{1}{12 \pi} f^{\frac{1}{2}} \partial_{x^{+}}^{2} f^{-\frac{1}{2}} .
$$

Combining eq. (A.18) and eq. (A.19), we get

$$
\left\langle X\left|T_{x^{+} x^{+}}\right| X\right\rangle=\left\langle x\left|T_{x^{+} x^{+}}\right| x\right\rangle-\frac{1}{24 \pi} \operatorname{Sch}\left(X^{+}, x^{+}\right) .
$$

There is an analogous formula with $\left(X^{+}, x^{+}\right)$being replaced by $\left(X^{-}, x^{-}\right)$. 


\section{B ADM mass}

In this appendix, we give the details of the computation of the ADM mass. Following the the standard holographic renormalisation methods (see, for instance, [151-159]), the ADM mass can be computed from the boundary stress tensor obtained by varying the action with respect to the boundary metric. The boundary is specified by a fixed value of dilaton, eq. (2.7). In Poincaré coordinates eq. (2.4), this in general will correspond to some trajectory $(t, z(t))$.

To compute the ADM mass, it is convenient to work in the Fefferman-Graham (FG) coordinates which we denote by $(\hat{t}, \hat{z})$. The metric in these coordinates takes the form

$$
\mathrm{d} s^{2}=g_{\hat{t} \hat{t}} \mathrm{~d} \hat{t}^{2}+\frac{\mathrm{d} \hat{z}^{2}}{\hat{z}^{2}}
$$

Asymptotically, as $\hat{z} \rightarrow 0$, the metric and dilation satisfy the conditions,

$$
\begin{aligned}
g_{\hat{t} \hat{t}} & =\frac{1}{\hat{z}^{2}}\left(1+\mathcal{O}\left(\hat{z}^{2}\right)\right) . \\
\phi & =\frac{1}{\mathcal{J} \hat{z}}+\mathcal{O}\left(\hat{z}^{0}\right) .
\end{aligned}
$$

\section{B.1 Fefferman-Graham coordinate transformation}

Let the coordinate transformation from the Poincaré coordinates to the FG coordinates be denoted by,

$$
\begin{aligned}
t & =H(\hat{t})+\hat{z}^{2} G(\hat{t})+\cdots, \\
z & =\hat{z} K(\hat{t})\left(1+\hat{z}^{2} J(\hat{t})+\cdots\right),
\end{aligned}
$$

where $H, G, J, K$ are functions which will be determined by imposing FG gauge and also by requiring that the boundary, to leading order, corresponds to constant $\hat{z}$. For ease of notation, the arguments of the functions $G, H, J, K$ will not be written explicitly in the rest of the discussion and it is understood that they are only functions of the time $\hat{t}$. In this appendix the derivatives with respect to $t$ will be denoted by primes and derivatives with respect to $\hat{t}$ will be denoted by dots. Also, we set $L_{2}=1$.

For the kind of situations we consider, it follows from the equations of motion that the general form of $\phi$ near the boundary in Poincaré coordinates is given by

$$
\phi=\frac{\zeta}{4}+\frac{f_{0}(t)}{z}+f_{2}(t) z+\mathcal{O}\left(z^{2}\right) .
$$

(In the classical case discussed in section 2, the first term on the r.h.s. is absent).

Expanding in FG coordinates, we get

$$
\phi=\frac{\zeta}{4}+\left.\frac{f_{0}(t)}{K \hat{z}}\right|_{t=H}+\left.\hat{z}\left(\frac{G f_{0}^{\prime}(t)-J f_{0}(t)}{K}+K f_{2}(t)\right)\right|_{t=H}+\mathcal{O}\left(\hat{z}^{2}\right) .
$$

Requiring that the dilaton goes like $\frac{1}{\mathcal{J} \hat{z}}$ to leading order fixes $K$ to be

$$
K=\mathcal{J} f_{0}(t) .
$$


Note that in eqs. (B.6) and (B.7), the coordinate $t$ in $f_{0}(t), f_{2}(t)$ has to be treated as a function of $\hat{t}$, i.e., $t=H(\hat{t})$. The metric under the transformation eq. (B.4) becomes,

$$
\begin{aligned}
\mathrm{d} s^{2}= & \left(-\frac{\dot{H}^{2}}{\hat{z}^{2} K^{2}}+\frac{2 J \dot{H}^{2}+\dot{K}^{2}-2 \dot{G} \dot{H}}{K^{2}}\right) \mathrm{d} \hat{t}^{2} \\
& +\left(\frac{1}{\hat{z}^{2}}+4 J-\frac{4 G^{2}}{K^{2}}\right) \mathrm{d} \hat{z}^{2}+\left(\frac{-4 G \dot{H}+2 K \dot{K}}{\hat{z} K^{2}}\right) \mathrm{d} \hat{t} \mathrm{~d} \hat{z} .
\end{aligned}
$$

Imposing the FG gauge, eqs. (B.1), (B.2), we obtain

$$
\begin{aligned}
\dot{H} & =K=\mathcal{J} f_{0}(t), \\
G & =\frac{K \dot{K}}{2 \dot{H}}=\frac{K K^{\prime}}{2}=\mathcal{J}^{2} \frac{f_{0} f_{0}^{\prime}}{2}, \\
J & =\frac{G^{2}}{K^{2}}=\mathcal{J}^{2} \frac{f_{0}^{\prime 2}}{4} .
\end{aligned}
$$

Using eqs. (B.7), (B.9)-(B.11), the dilaton (B.6) and the metric (B.8) become

$$
\begin{aligned}
\phi & =\frac{\zeta}{4}+\frac{1}{\mathcal{J} \hat{z}}+\mathcal{J} \hat{z}\left(\frac{f_{0}^{\prime 2}}{4}+f_{0} f_{2}\right)+\mathcal{O}\left(\hat{z}^{2}\right), \\
\mathrm{d} s^{2} & =-\left(\frac{1}{\hat{z}^{2}}-\mathcal{J}^{2}\left(\frac{f_{0}^{\prime 2}}{2}-f_{0} f_{0}^{\prime \prime}\right)+\mathcal{O}\left(\hat{z}^{2}\right)\right) \mathrm{d} \hat{t}^{2}+\left(\frac{1}{\hat{z}^{2}}+\mathcal{O}\left(\hat{z}^{2}\right)\right) \mathrm{d} \hat{z}^{2}+\mathcal{O}(\hat{z}) \mathrm{d} \hat{z} \mathrm{~d} \hat{t} .
\end{aligned}
$$

It is to be noted that $t=H(\hat{t})$ in eq. (B.9)-eq. (B.13). We also note that the coordinate $\hat{t}$ is obtained from $t$ as follows. Using eq. (B.9) we can obtain $\hat{t}$ in terms of $H$ by solving the equation

$$
\frac{1}{\mathcal{J}} \int \frac{\mathrm{d} H}{f_{0}(H)}=\hat{t}
$$

From eq. (B.9) we see that this gives $\hat{t}$ in terms of $t$.

\section{B.2 Classical mass}

The ADM mass is defined as,

$$
M=\lim _{\hat{z} \rightarrow \hat{z}_{B}} \frac{-2 \hat{z}}{\sqrt{-\gamma}}\left(\frac{\delta I}{\delta \gamma^{\hat{t} \hat{t}}}\right)
$$

where $\hat{z}_{B}$ is the boundary value of $\hat{z}$ eq. (2.7), i.e., $\phi_{B}=\frac{1}{\mathcal{J} \hat{z}_{B}}+\mathcal{O}\left(\hat{z}_{B}^{0}\right)$, and $\gamma_{\mu \nu}$ refers to the boundary metric. For the classical case, $I$ is the JT action given in eq. (2.1). After some calculations, this gives

$$
M_{J T}=\frac{-1}{8 \pi G} \lim _{\hat{z} \rightarrow \hat{z}_{B}} \hat{z} \gamma_{\hat{t} \hat{t}}\left(\hat{z} \partial_{\hat{z}} \phi+\phi\right) .
$$

Using the form of the dilaton, eq. (B.12), and the metric eq. (B.13), the mass eq. (B.16) becomes

$$
M_{J T}=\frac{\mathcal{J}}{4 \pi G}\left(\frac{f_{0}^{\prime 2}}{4}+f_{0} f_{2}\right)+\mathcal{O}\left(\hat{z}_{B}\right) .
$$


Comparing the general vacuum solution for the dilaton, eq. (2.8), with the form eq. (B.5) (without the term $\frac{\zeta}{4}$ ), we get

$$
f_{0}=\frac{1}{\mathcal{J}}\left(\frac{a}{2}+b t+\frac{c t^{2}}{2}\right), f_{2}=-\frac{c}{2 \mathcal{J}}
$$

Therefore using eq. (B.17) and eq. (B.18), we get mass for the vacuum solution to be eq. (2.11).

For a general solution for the dilaton given in eq. (2.30), one can derive an expression for the ADM mass in terms of the value of $h$ at the boundary. We expand $h\left(x^{-}\right)$in a Taylor series in $z$ near the boundary to obtain,

$$
\phi=\frac{h(t)}{2 z}-\frac{h^{\prime \prime}(t)}{4} z+\mathcal{O}\left(z^{2}\right) .
$$

By comparing eq. (B.5) and (B.19), we note that

$$
f_{0}=\frac{h(t)}{2}, \quad f_{2}=-\frac{h^{\prime \prime}(t)}{4}
$$

Expressed in terms of FG coordinates, the dilaton near the boundary takes the form (B.12) (without the term $\frac{\zeta}{4}$ ),

$$
\phi=\frac{1}{\mathcal{J} \hat{z}}+\frac{1}{16}\left(h^{2}-2 h h^{\prime \prime}\right) \mathcal{J} \hat{z}+\cdots .
$$

From eq. (B.17) and eq. (B.20), we obtain the classical mass in terms of $h$ as

$$
M=\frac{\mathcal{J}}{64 \pi G}\left(h^{\prime 2}-2 h h^{\prime \prime}\right) .
$$

From the discussion in section B.1, we have seen that asymptotically $t=H(\hat{t})$ and therefore from eq. (B.9) and eq. (B.4),

$$
\frac{\mathrm{d} t}{\mathrm{~d} \hat{t}}=\frac{\mathcal{J} h(t)}{2} .
$$

These observations allow the mass to be expressed as

$$
M=-\frac{1}{8 \pi G \mathcal{J}} \operatorname{Sch}(t, \hat{t})
$$

where the Schwarzian derivative on the r.h.s. is given by eq. (2.14). We also note that $\hat{t}$ can be taken to be the proper time along the boundary (after suitably rescaling by $z_{B}$ ), therefore the Schwarzian derivative is with respect to the proper time on the boundary.

It follows from eq. (B.15) that the mass formula derived above has corrections which are of fractional order $\hat{z}_{B} T$ where $T \sim \sqrt{\mu}$ is the temperature. These are small when $\hat{z}_{B} T \ll 1$. Using eq. (B.3) this condition can be expressed as eq. (2.20). Similarly the mass for the $\chi$ and $\psi$ systems will also be calculated below in the limit eq. (2.20). 


\section{B.3 Mass for the $\chi$ system}

With the general form of the dilaton given in eq. (B.5) and the form of the solution for $\chi$, eq. (3.29), it is straightforward to see that general form of the dilaton and $\chi$ near the boundary in FG coordinates is given by,

$$
\begin{aligned}
& \phi=\frac{\alpha_{-1}(\hat{t})}{\hat{z}}+\frac{\zeta}{4}+\alpha_{1}(\hat{t}) \hat{z}+\alpha_{2}(\hat{t}) \hat{z}^{2}+\cdots, \\
& \chi=\ln \hat{z}+\sigma_{0}(\hat{t})+\sigma_{1}(\hat{t}) \hat{z}+\sigma_{2}(\hat{t}) \hat{z}^{2}+\cdots,
\end{aligned}
$$

for some functions $\alpha_{i}(\hat{t})$ and $\sigma_{i}(\hat{t})$. For the system with $\chi$ field, the action $I$ in eq. (B.15) is given by

$$
I=I_{J T}+I_{\chi}+I_{\mathrm{ct}} .
$$

Here $I_{J T}$ is the JT action given in eq. (2.1), and $I_{\chi}$ is the action for the $\chi$ field defined in eq. (3.3). $I_{\mathrm{ct}}$ is a counterterm which has to be added to cancel the divergences and is given by

$$
I_{\mathrm{ct}}=-\frac{N}{24 \pi} \int_{\mathrm{bdy}} \sqrt{-\gamma}
$$

The mass becomes

$$
\begin{aligned}
M & =-\lim _{\hat{z} \rightarrow \hat{z}_{B}} \hat{z} \gamma_{\hat{t} \hat{t}}\left(\frac{1}{8 \pi G}\left(\hat{z} \partial_{\hat{z}} \phi+\phi\right)-\frac{N}{12 \pi}\left(\hat{z} \partial_{\hat{z} \chi}-\frac{1}{2}\right)\right) \\
& =\left(\frac{\alpha_{1}(\hat{t})}{4 \pi G}-\frac{N \sigma_{1}(\hat{t})}{12 \pi}\right)+\mathcal{O}\left(\hat{z}_{B}\right) .
\end{aligned}
$$

Using this we will now derive the formulae for the mass in the cases of eternal black hole and infalling matter. For an eternal black hole, the metric in Schwarzschild coordinates is given by eq. (3.4) and the dilaton is given in eq. (3.16). In Poincaré coordinates, the dilaton becomes eq. (A.2). Also, the value of $\chi$ for an eternal black hole is given by eq. (3.51). Converting the dilaton and $\chi$ to FG coordinates and Taylor expanding in powers of $\hat{z}$ near the boundary gives

$$
\begin{aligned}
\phi & =\frac{1}{\mathcal{J} \hat{z}}+\frac{\zeta}{4}+\frac{\mu}{4 \mathcal{J}} \hat{z}+\cdots \\
\chi & =\ln \hat{z}+\ln \left(\tilde{\phi}_{B} \mathcal{J}\right)-\sqrt{\mu} \hat{z}+\cdots .
\end{aligned}
$$

Therefore, from eqs. (B.30), (B.31), (B.29), we get the mass of the eternal black hole to be eq. (3.24).

For the case of an infalling matter pulse in this system, the classical mass eq. (2.38) gets a correction due to the contribution from the $\chi$ field. We expand the solution eq. (3.41) near the boundary in $\hat{z}$ coordinate,

$$
\chi=\ln \left(\frac{\hat{z} \mathcal{J} h}{2}\right)+\ln \left(\frac{2 \tilde{\phi}_{B}-h^{\prime}}{h}\right)+\left(\frac{h h^{\prime \prime}}{2\left(2 \tilde{\phi}_{B}-h^{\prime}\right)}+\frac{h^{\prime}}{2}\right) \mathcal{J} \hat{z}+\mathcal{O}\left(\hat{z}^{2}\right) .
$$


Using eq. (B.29) and eq. (B.32), and taking the limit eq. (3.43), we obtain the mass to be

$$
M_{\chi}=\frac{\mathcal{J}}{64 \pi G}\left(h^{\prime 2}-2 h h^{\prime \prime}\right)-\frac{N \mathcal{J} h^{\prime}}{24 \pi} .
$$

We can extend the above calculation to the general case where $\chi$ field is given by eq. (3.61). The function $f_{-}\left(x^{-}\right)$in eq. (3.61) is determined by imposing Dirichlet boundary condition on $\chi$ at the boundary. Doing so gives $\chi$ as

$$
\chi=\ln \left(\frac{x^{+}-x^{-}}{2}\right)-\ln \left(1+a x^{+}\right)-\ln \left(\frac{\frac{h\left(x^{-}\right)}{2}}{\tilde{\phi}_{B}-\frac{h^{\prime}\left(x^{-}\right)}{2}}\right)+\ln \left(1+a x^{-}+\frac{a h\left(x^{-}\right)}{\tilde{\phi}_{B}-\frac{h^{\prime}\left(x^{-}\right)}{2}}\right) .
$$

Expanding the above solution for $\chi$ in FG $\hat{z}$ coordinates near the boundary we get

$$
\begin{aligned}
\chi= & \ln \frac{\hat{z} \mathcal{J} h}{2}+g_{0}(\hat{t}) \\
& +\left(\frac{h^{\prime}}{2}-\frac{a h}{2(1+a t)}+\frac{(1+a t) h h^{\prime \prime}-2 a h\left(\phi_{B}-\frac{h^{\prime}}{2}\right)-2 a h h^{\prime}}{4\left(a h+(1+a t)\left(\tilde{\phi}_{B}-\frac{h^{\prime}}{2}\right)\right)}\right) \mathcal{J} \hat{z}+\mathcal{O}\left(\hat{z}^{2}\right),
\end{aligned}
$$

where $g_{0}(\hat{t})$ is a function which is unimportant for the calculation of mass. Using eq. (B.21) (with the additional quantum term, $\frac{\zeta}{4}$ as in (B.5)), eq. (B.35), the expression for the mass (B.29) in the limit eq. (3.43) becomes

$$
M_{\chi}=\frac{\mathcal{J}}{64 \pi G}\left(h^{\prime 2}-2 h h^{\prime \prime}\right)-\frac{N \mathcal{J}}{12 \pi}\left(\frac{h^{\prime}}{2}-\frac{a h}{a t+1}\right) .
$$

Writing the above expression for the mass in terms of the $t(\hat{t})$ using eq. (B.23), we get

$$
M_{\chi}=-\frac{1}{8 \pi G \mathcal{J}} \operatorname{Sch}(t, \hat{t})-\frac{N}{12 \pi}\left(\frac{t^{\prime \prime}}{t^{\prime}}-\frac{2 a t^{\prime}}{a t+1}\right) .
$$

The above formula for mass is an $\operatorname{SL}(2, R)$ invariant quantity. This can be understood as follows. Under an $\mathrm{SL}(2, R)$ transformation, eq. (3.59) it follows from eq. (B.23) and eq. (3.60) (along with the fact that $\hat{t}$ does not transform under $\operatorname{SL}(2, R)$ ) that

$$
t(\hat{t}) \rightarrow \frac{p t(\hat{t})+q}{r t(\hat{t})+s}
$$

It is then straightforward to verify that the expression for mass eq. (B.37) is invariant when $t(\hat{t})$ and $a$ also transform as given in eq. (B.38) and eq. (3.63) respectively.

\section{B.4 Mass for $\psi$ system}

To compute the ADM mass for the $\psi$ system, we once again use the definition eq. (B.15) with $I$ given by

$$
I=I_{J T}+I_{\psi}+I_{\mathrm{ct}},
$$


where $I_{J T}$ is the JT action, eq. (2.1), $I_{\psi}$ is the action for the matter fields $\psi_{i}$, eq. (1.2), and $I_{\mathrm{ct}}$ is the counter term action, which is the same in the $\chi$ system, eq. (B.28). To obtain the quantum correction from $I_{\psi}$, we can examine the conformal anomaly, eq. (4.6), for the $N$ scalar fields $\psi_{i}$ but now including the boundary contribution. It is given by $[160,161]$

$$
T_{\mu}^{\mu}=\frac{N}{24 \pi}\left(R+2 K \delta\left(x_{\perp}\right)\right)
$$

where $K$ is the extrinsic trace of the boundary. Therefore from the definition of the stress tensor,

$$
T_{\mu \nu}^{\psi}=-\frac{2}{\sqrt{-g}} \frac{\delta I_{\psi}}{\delta g^{\mu \nu}},
$$

we can obtain the contribution to the mass from $I_{\psi}$ as follows,

$$
\begin{aligned}
\lim _{\hat{z} \rightarrow \hat{z}_{B}} \frac{-2 \hat{z}}{\sqrt{-\gamma}}\left(\frac{\delta I_{\psi}}{\delta \gamma^{\hat{t} \hat{t}}}\right) & =\left.\lim _{\hat{z} \rightarrow \hat{z}_{B}} \hat{z} T_{\hat{t} \hat{t}}\right|_{\text {bdy }} \\
& =\frac{N}{12 \pi} \lim _{\hat{z} \rightarrow \hat{z}_{B}} \hat{z} \gamma_{\hat{t} \hat{t}} K .
\end{aligned}
$$

Therefore using eq. (B.15), (B.39), (B.16), (B.12) (B.42) and eq. (B.28), the expression for the mass for the $\psi$ system becomes

$$
\begin{aligned}
M_{\psi} & =-\lim _{\hat{z} \rightarrow \hat{z}_{B}} \hat{z} \gamma_{\hat{t} \hat{t}}\left(\frac{1}{8 \pi G}\left(\hat{z} \partial_{\hat{z}} \phi+\phi\right)-\frac{N}{12 \pi}\left(K-\frac{1}{2}\right)\right) \\
& =\frac{1}{4 \pi G}\left(f_{0} f_{2}+\frac{f_{0}^{\prime 2}}{4}\right)-\frac{N}{12 \pi} \lim _{\hat{z} \rightarrow \hat{z}_{B}} \frac{1}{\hat{z}}(K-1) .
\end{aligned}
$$

From eq. (B.13) it can be easily shown that the second term on the r.h.s. above is given by

$$
\lim _{\hat{z} \rightarrow \hat{z}_{B}} \frac{1}{\hat{z}}(K-1)=\mathcal{J}^{2} \hat{z}_{B}\left(\frac{f_{0}^{\prime 2}}{2}-f_{0} f_{0}^{\prime \prime}\right),
$$

which involves the same function as appears in the first term in eq. (B.43), when expressed in terms of $h$ using eq. (B.20). Thus we see that the quantum effects simply serve to change the coefficient in front of the mass compared to the classical case; this can be incorporated by rescaling $G$, eq. (4.19).

It follows then from eq. (B.43) that the mass of an eternal black holes is given by

$$
M_{\psi}=\frac{1}{16 \pi G \mathcal{J}}\left(1-\frac{\zeta}{2 \tilde{\phi}_{B}}\right) \mu,
$$

and the mass in the presence of infalling matter is given by

$$
M_{\psi}=\frac{\mathcal{J}}{64 \pi G}\left(1-\frac{\zeta}{2 \tilde{\phi}_{B}}\right)\left(h^{\prime 2}-2 h h^{\prime \prime}\right) .
$$

From B.2 we see that the mass can be expressed in terms of the Schwarzian, eq. (B.24) with $G$ rescaled as given in (4.19). 


\section{Late time behaviour of $h$}

In this appendix, we consider the $\chi$ system with infalling matter satisfying the condition (2.26) and show that the late time behaviour, after matter has stopped falling in, is given by eq. (3.77). We also show by an explicit coordinate transformation that the late time behaviour eq. (3.66) corresponds to a black hole of the correct mass.

We first present the arguments for the case of system initially being in the Poincaré vacuum. We start at $x^{-}=0$ with $\phi$ given by eq. (3.34) which corresponds to eq. (3.37) in eq. (3.36), i.e.,

$$
h(0)=\frac{1}{\mathcal{J}}, h^{\prime}(0)=0, h^{\prime \prime}(0)=0,
$$

and $\chi$ is given by eq. (3.49) We consider situations where the matter starts falling in at $x^{-}=0$, and stops falling in at say $x^{-}=x_{f}^{-}$. We will argue below that while the matter is falling in $h$ decreases monotonically and consider situations where $h$ continues to be positive until the instant $x_{f}^{-}$. We then argue that for $x^{-}>x_{f}^{-}, h$ continues to decrease and eventually hits a first order zero at say $x_{0}$, in whose vicinity it takes the form, eq. (3.77).

To show that while the matter is falling in $h$ decreases monotonically we note the following. At $x^{-}=0, h^{\prime \prime \prime}<0$, while $h^{\prime}=h^{\prime \prime}=0$ from eq. (C.1). This means initially, near $x^{-}=0, h^{\prime}<0$ and $h^{\prime \prime}<0$. Next it can be argued that the function $h^{\prime}$ cannot have a minimum. We have noted that initially $h^{\prime \prime}<0$, for it to change sign it would have to go through zero. However we see from eq. (3.45) that when $h^{\prime \prime}=0, h^{\prime \prime \prime}<0$, thus even if $h^{\prime \prime}$ were to hit zero, it would subsequently only decrease and therefore stay negative. Since $h^{\prime \prime}<0$ it follows that $h^{\prime}$ stays negative starting from its initially negative value.

Next we will analyse the subsequent behaviour after the matter stops falling in at $x_{f}^{-}$. Before proceeding however, let us make the following observation. If the infalling matter falls in over a time scale $\tau$ then since $|h| \leq \frac{1}{\mathcal{J}}$ the condition eq. (3.43) is met if

$$
\frac{1}{\tau \mathcal{J} \phi_{\mathcal{B}}} \ll 1
$$

We are taking the infalling matter to obey this condition so that eq. (3.43) is valid.

Once matter stops falling in $h$ satisfies,

$$
h^{\prime 2}-2 h h^{\prime \prime}-2 \zeta h^{\prime}=\frac{64 \pi G M}{\mathcal{J}},
$$

for a constant value of the mass $M>0$, see eq. (3.47).

To simplify eq. (C.3) we introduce the variables $\tau$ and $y$ defined by

$$
\frac{\mathrm{d}}{\mathrm{d} \tau}=h \frac{\mathrm{d}}{\mathrm{d} x^{-}}, \quad y=\frac{\mathrm{d}}{\mathrm{d} \tau} \ln h=\frac{\mathrm{d} h}{\mathrm{~d} x^{-}} .
$$

It can be shown that $\tau$ is proportional to the proper time at the boundary, see appendix B. Rewriting eq. (C.3) in terms of $y$ and $\tau$ using eq. (C.4), we get

$$
\frac{\mathrm{d} y}{\mathrm{~d} \tau}-\frac{y^{2}}{2}+\zeta y=-\tilde{M},
$$


where we have defined

$$
\tilde{M}=32 \pi G \frac{M}{\mathcal{J}}
$$

The two roots of the equation

$$
y^{2}-2 \zeta y-2 \tilde{M}=0
$$

are

$$
\begin{aligned}
& y_{1}=\zeta-\sqrt{\zeta^{2}+2 \tilde{M}} \\
& y_{2}=\zeta+\sqrt{\zeta^{2}+2 \tilde{M}}
\end{aligned}
$$

we see that $y_{1}<0$ and $y_{2}>0$. Initially, since we have argued above that $h^{\prime \prime}<0$, it follows from eq. (C.4) that right after the matter stops falling in, at $x^{-}=x_{f}^{-}, \frac{d y}{d \tau}<0$. Thus the value $y$ takes at this time which we denote by $y_{f}$ satisfies the condition $y_{1}<y_{f}<y_{2}$. It then follows from eq. (C.5) that subsequently $y$ decreases and ultimately reaches $y_{1}$ with the proper time elapsed being proportional to,

$$
\Delta \tau(y)=-\int_{y}^{y_{f}} \frac{2}{y^{2}-2 \zeta y-2 \tilde{M}} \mathrm{~d} y .
$$

From eqs. (C.4) and (C.10), we can obtain $h$ and $x^{-}$in terms of $y$ as

$$
\begin{aligned}
h & =h_{0} e^{U} \quad \text { where } U=\int \frac{2 y}{y^{2}-2 \eta y-2 \tilde{M}} \mathrm{~d} y, \\
x^{-} & =x_{c}+\int \frac{2 h_{0} e^{U}}{y^{2}-2 \zeta y-2 \tilde{M}} \mathrm{~d} y,
\end{aligned}
$$

where $h_{0}$ and $x_{c}$ are integration constants which depend on the values of $h, h^{\prime}$ and $h^{\prime \prime}$ at $x_{f}^{-}$.

In the vicinity of $y=y_{1}$ we get from eq. (C.11) that

$$
U=\beta_{0}+\beta_{1} \ln \left(y-y_{1}\right)+\beta_{2}\left(y-y_{1}\right)+\cdots,
$$

where $\beta_{0}, \beta_{1}$ and $\beta_{2}$ are constants independent of $y$,

$$
\beta_{0}=-\frac{2 y_{2}}{y_{1}-y_{2}} \ln \left(y_{2}-y_{1}\right), \beta_{1}=\frac{2 y_{1}}{y_{1}-y_{2}}, \beta_{2}=-\frac{2 y_{2}}{\left(y_{1}-y_{2}\right)^{2}} .
$$

Using eq. (C.13) in eq. (C.11), eq. (C.12), we get

$$
\begin{aligned}
\left(x_{0}-x^{-}\right) & =\frac{2 h_{0} e^{\beta_{0}}}{\beta_{1}\left(y_{2}-y_{1}\right)}\left(y-y_{1}\right)^{\beta_{1}}\left(1+\frac{\beta_{1}}{\beta_{1}+1}\left(\beta_{2}-\frac{1}{y_{1}-y_{2}}\right)\left(y-y_{1}\right)\right), \\
h & =h_{0} e^{\beta_{0}}\left(y-y_{1}\right)^{\beta_{1}}\left(1+\beta_{2}\left(y-y_{1}\right)+\mathcal{O}\left(\left(y-y_{1}\right)^{2}\right)\right),
\end{aligned}
$$

where $x^{-} \rightarrow x_{0}$ when $y \rightarrow y_{1}$. Eq. (C.15) and (C.16) give

$$
h=\tilde{c}_{1}\left(x_{0}-x^{-}\right)+\tilde{c}_{2}\left(x_{0}-x^{-}\right)^{2+\alpha},
$$


where

$$
\tilde{c}_{1}=-y_{1}
$$

and

$$
\alpha=-\frac{\zeta}{y_{1}}=\frac{\zeta}{\tilde{c}_{1}} .
$$

The form of $h$ in terms of $x^{-}$,eq. (C.17) and the value of the exponent $\alpha$ in eq. (C.19) agrees with eq. (3.77) and eq. (3.74) respectively, when $\tilde{c}_{1}$ is identified with $\frac{c_{1}}{\mathcal{J}}$ in eq. (3.77). The value of $\tilde{c}_{2}$ in eq. (C.17) depends on $h_{0}$ and is unimportant for our discussion.

Let us end this discussion with some comments regarding the case where the system starts from a black hole configuration which corresponds to taking $\chi$ as eq. (3.51) and $\phi$ as in eq. (3.50). The arguments following eq. (C.1) showing the monotonic decrease of $h$ as matter falls, can be extended to this case in a straightforward manner.

As matter falls in, $h$ satisfies the equation eq. (3.57), with the initial conditions

$$
h(0)=\frac{1}{\mathcal{J}}, h^{\prime}(0)=0, h^{\prime \prime}(0)=-\frac{2 \mu}{\mathcal{J}} .
$$

From eq. (3.57) and eq. (C.20) it can be seen that $h^{\prime \prime \prime}<0$ at $x^{-}=0$. Therfore $h^{\prime \prime}$ continues to decrease starting from the initial value in eq. (C.20). It is straightforward to see from eq. (3.57) that when $h^{\prime \prime}=0, h^{\prime \prime \prime}<0$ and therefore $h^{\prime \prime}$ would subsequently decrease as in the case of Poincaré vacuum initial condition. Thus $h^{\prime}$ is always negative and therefore $h$ decreases monotonically.

In the discussion above we have seen that $h$ monotonically decreases. This self consistently justifies neglecting the terms with additional time derivatives in eq. (3.42). Each term with an extra derivative in eq. (3.42) is also accompanied with an extra power of $h$ on dimensional grounds. Since $h=\frac{1}{\mathcal{J}}$ at $x^{-}=0$, when the matter starts falling in, and decreases subsequently, the condition, eq. (3.43) suppresses any term with an extra derivative. Similarly, we see that given eq. (2.20) the monotonic decrease of $h$ also leads to eq. (3.56) being valid, and along with eq. (3.43) then leads to eq. (3.55) being well-approximated by eq. (3.57).

\section{C.1 Late time black hole coordinates}

Here, we show that the late time form of $h$ given in eq. (3.66) corresponds to a black hole with mass $M$, eq. (3.24) with $\mu=\frac{c_{1}^{2}}{4}$.

From eq. (3.66) and using eq. (3.36), the dilaton at late times is given by

$$
\phi=\frac{1}{\mathcal{J}}\left(\frac{c_{1}\left(x_{0}-x^{-}\right)}{x^{+}-x^{-}}-\frac{1}{2} c_{1}\right)+\frac{\zeta}{4} .
$$

By taking

$$
c_{1} \rightarrow 2 \sqrt{\mu}, x_{0} \rightarrow \frac{1}{\sqrt{\mu}}, x^{ \pm} \rightarrow \frac{2 x^{ \pm}}{\sqrt{\mu} x^{ \pm}+1},
$$

the solution eq. (C.21) can be converted to the standard form for the black hole solution

$$
\phi=\frac{1}{\mathcal{J}}\left(\frac{1-\mu x^{+} x^{-}}{x^{+}-x^{-}}\right)+\frac{\zeta}{4},
$$


which is obtained by taking

$$
h=\frac{1}{\mathcal{J}}\left(1-\mu\left(x^{-}\right)^{2}\right)
$$

in eq. (3.36).

The coordinate transformation relating Schwarzschild coordinates $t_{s}, r$ with $x^{ \pm}$in eq. (C.21) is

$$
\begin{aligned}
r & =\frac{c_{1}\left(x_{0}-x^{-}\right)}{x^{+}-x^{-}}-\frac{1}{2} c_{1} \\
t_{s} & =-\frac{1}{c_{1}} \ln \left(\left(x_{0}-x^{-}\right)\left(x_{0}-x^{+}\right)\right) .
\end{aligned}
$$

This gives for the metric,

$$
\mathrm{d} s^{2}=-\frac{4}{\left(x^{+}-x^{-}\right)^{2}} \mathrm{~d} x^{+} \mathrm{d} x^{-}=-\left(r^{2}-\frac{c_{1}^{2}}{4}\right) \mathrm{d} t_{s}^{2}+\frac{\mathrm{d} r^{2}}{\left(r^{2}-\frac{c_{1}^{2}}{4}\right)} .
$$

Comparing with eq. (3.4) we see that this is a black hole of mass $M$, eq. (3.24) with the identification eq. (3.67). The temperature of the black hole eq. (C.26) is also easily seen to be

$$
T=\frac{c_{1}}{4 \pi}
$$

\section{Second law violation for apparent horizon}

In this appendix, we consider the $\chi$ system and show by taking an explicit example for $T_{--}^{m}$ corresponding to a delta-function pulse that the generalized second law is violated when the generalized entropy is computed along the classical apparent horizon, eq. (2.45).

We take the matter stress tensor corresponding to infalling matter to be

$$
T_{--}^{m}=\tilde{\mu} \delta\left(x^{-}\right) \text {for } \tilde{\mu}>0 .
$$

Before the pulse went in, the geometry is pure $A d S_{2}$, eq. (2.4), and we take the dilaton to be specified by eq. (3.34), which corresponds to $h=\frac{1}{\mathcal{J}}$. Although the equation for $h$, eq. (3.45), can be solved exactly for the matter stress tensor, eq. (D.1), as we saw above in appendix $\mathrm{C}$, we resort to a perturbative expansion in $\zeta$ for simplicity. The total solution for $h$ can be written as

$$
h=h^{(0)}+\zeta h^{(1)}+\mathcal{O}\left(\zeta^{2}\right) .
$$

The zeroth order solution $h^{(0)}$ is obtained by integrating (3.45) with the stress tensor eq. (D.1) and setting $\zeta=0$ which gives

$$
h^{(0)}=\frac{1}{\mathcal{J}}\left(1-\mu\left(x^{-}\right)^{2}\right),
$$

where

$$
\mu=8 \pi G \tilde{\mu} \mathcal{J} .
$$


Expanding eq. (3.45) to $\mathcal{O}(\zeta)$, we get the equation for $h^{(1)}$ as

$$
h^{(1) \prime \prime \prime}=-\frac{h^{(0) \prime \prime}}{h^{(0)}} .
$$

Using the solution eq. (D.3), we can solve for $h^{(1)}$ to get

$$
h^{(1)}=-\frac{1}{2 \sqrt{\mu}}\left(2 \sqrt{\mu} x^{-}+\left(1-\sqrt{\mu} x^{-}\right)^{2} \ln \left(1-\sqrt{\mu} x^{-}\right)-\left(1+\sqrt{\mu} x^{-}\right)^{2} \ln \left(1+\sqrt{\mu} x^{-}\right)\right),
$$

The perturbative solution is a good approximation everywhere except at late times near $x^{-}=\frac{1}{\sqrt{\mu}}$ where $h^{(0)}$ goes to zero. The trajectory of the apparent horizon gets corrected due to the correction in $h$. From the general form of the trajectory of the apparent horizon,eq. (2.46), and using eq. (D.3), eq. (D.6), we get the apparent horizon trajectory to $\mathcal{O}(\zeta)$ to be

$$
\left.x^{+}\right|_{\mathrm{ah}}=\frac{1}{\sqrt{\mu}}-\frac{\mathcal{J} \zeta}{2 \mu}\left(\sqrt{\mu} x^{-}-2 \ln \left(1+\sqrt{\mu} x^{-}\right)\right) .
$$

The dilaton, (3.36), and $\chi$, eq. (3.41) at the apparent horizon to $\mathcal{O}(\zeta)$, using the solutions eq. (D.3), eq. (D.6), become

$$
\begin{aligned}
\left.\phi\right|_{\mathrm{ah}} & =\frac{\sqrt{\mu}}{\mathcal{J}}+\frac{\zeta}{4}\left(1-2 \sqrt{\mu} x^{-}\right), \\
\left.\chi\right|_{\mathrm{ah}} & =-\ln \left(\frac{\sqrt{\mu}}{\mathcal{J}}\left(1+\sqrt{\mu} x^{-}\right)\right)+O(\zeta) .
\end{aligned}
$$

Using eq. (D.8) and eq. (D.9), the entropy at the apparent horizon is given by

$$
\begin{aligned}
S_{\text {gen }}^{\chi} & =\left.\left(\frac{\phi}{4 G}-\frac{N \chi}{6}\right)\right|_{\mathrm{ah}} \\
& =\frac{1}{4 G}\left(\frac{\sqrt{\mu}}{\mathcal{J}}+\frac{\zeta}{2}\left(\frac{1}{2}-\sqrt{\mu} x^{-}+\ln \left(\frac{\sqrt{\mu}}{\mathcal{J}}\left(1+\sqrt{\mu} x^{-}\right)\right)\right)+\mathcal{O}\left(\zeta^{2}\right)\right) .
\end{aligned}
$$

Ignoring the $\mathcal{O}\left(\zeta^{2}\right)$ correction, it is clear from the above expression that entropy is in fact a monotonically decreasing function of $x^{-}$and therefore the generalized second law is violated at the apparent horizon.

The same conclusion can be reached, without taking recourse to perturbation theory in $\zeta$, by solving eq. (3.45) numerically for the case of a delta function matter pulse with initial conditions as given in eq. (C.1).

Open Access. This article is distributed under the terms of the Creative Commons Attribution License (CC-BY 4.0), which permits any use, distribution and reproduction in any medium, provided the original author(s) and source are credited.

\section{References}

[1] R. Jackiw, Lower dimensional gravity, Nucl. Phys. B 252 (1985) 343. 
[2] C. Teitelboim, Gravitation and Hamiltonian structure in two space-time dimensions, Phys. Lett. B 126 (1983) 41.

[3] T. Muta and S.D. Odintsov, Two-dimensional higher derivative quantum gravity with constant curvature constraint, Prog. Theor. Phys. 90 (1993) 247 [InSPIRE].

[4] J.P.S. Lemos and P.M. Sa, Nonsingular constant curvature two-dimensional black hole, Mod. Phys. Lett. A 9 (1994) 771 [gr-qc/9309023] [InSPIRE].

[5] J.P.S. Lemos, Thermodynamics of the two-dimensional black hole in the Teitelboim-Jackiw theory, Phys. Rev. D 54 (1996) 6206 [gr-qc/9608016] [INSPIRE].

[6] A. Almheiri and J. Polchinski, Models of AdS 2 backreaction and holography, JHEP 11 (2015) 014 [arXiv: 1402.6334] [INSPIRE].

[7] K. Jensen, Chaos in AdS 2 holography, Phys. Rev. Lett. 117 (2016) 111601 [arXiv: 1605.06098] [INSPIRE].

[8] J. Engelsöy, T.G. Mertens and H. Verlinde, An investigation of $A d S_{2}$ backreaction and holography, JHEP 07 (2016) 139 [arXiv: 1606.03438] [INSPIRE].

[9] J. Maldacena, D. Stanford and Z. Yang, Conformal symmetry and its breaking in two dimensional nearly Anti-de-Sitter space, PTEP 2016 (2016) 12C104 [arXiv:1606.01857] [INSPIRE].

[10] M. Cvetič and I. Papadimitriou, AdS 2 holographic dictionary, JHEP 12 (2016) 008 [Erratum ibid. 01 (2017) 120] [arXiv: 1608.07018] [INSPIRE].

[11] J. Maldacena, D. Stanford and Z. Yang, Diving into traversable wormholes, Fortsch. Phys. 65 (2017) 1700034 [arXiv: 1704.05333] [INSPIRE].

[12] S. Dubovsky, V. Gorbenko and M. Mirbabayi, Asymptotic fragility, near AdS 2 holography and TT , JHEP 09 (2017) 136 [arXiv:1706.06604] [INSPIRE].

[13] M. Taylor, Generalized conformal structure, dilaton gravity and SYK, JHEP 01 (2018) 010 [arXiv:1706.07812] [INSPIRE].

[14] I. Kourkoulou and J. Maldacena, Pure states in the SYK model and nearly-AdS ${ }_{2}$ gravity, arXiv: 1707.02325 [INSPIRE].

[15] D. Grumiller et al., Menagerie of $A d S_{2}$ boundary conditions, JHEP 10 (2017) 203 [arXiv: 1708.08471] [INSPIRE].

[16] H.A. González, D. Grumiller and J. Salzer, Towards a bulk description of higher spin SYK, JHEP 05 (2018) 083 [arXiv:1802.01562] [INSPIRE].

[17] A. Gaikwad, L.K. Joshi, G. Mandal and S.R. Wadia, Holographic dual to charged SYK from $3 D$ gravity and Chern-Simons, JHEP 02 (2020) 033 [arXiv: 1802.07746] [InSPIRE].

[18] P. Nayak et al., On the dynamics of near-extremal black holes, JHEP 09 (2018) 048 [arXiv: 1802.09547] [INSPIRE].

[19] K.S. Kolekar and K. Narayan, AdS 2 dilaton gravity from reductions of some nonrelativistic theories, Phys. Rev. D 98 (2018) 046012 [arXiv: 1803.06827] [InSPIRE].

[20] J. Maldacena and X.-L. Qi, Eternal traversable wormhole, arXiv: 1804.00491 [INSPIRE].

[21] D. Harlow and D. Jafferis, The factorization problem in Jackiw-Teitelboim gravity, JHEP 02 (2020) 177 [arXiv: 1804.01081] [INSPIRE].

[22] Y.-Z. Li, S.-L. Li and H. Lü, Exact embeddings of JT gravity in strings and M-theory, Eur. Phys. J. C 78 (2018) 791 [arXiv:1804.09742] [InSPIRE]. 
[23] I. Bena, P. Heidmann and D. Turton, AdS 2 holography: mind the cap, JHEP 12 (2018) 028 [arXiv: 1806.02834 ] [INSPIRE].

[24] F. Larsen, A nAttractor mechanism for nAdS $S_{2} / n C F T_{1}$ holography, JHEP 04 (2019) 055 [arXiv: 1806.06330] [INSPIRE].

[25] A. Goel, H.T. Lam, G.J. Turiaci and H. Verlinde, Expanding the black hole interior: partially entangled thermal states in SYK, JHEP 02 (2019) 156 [arXiv:1807.03916] [INSPIRE].

[26] J. Lin, Entanglement entropy in Jackiw-Teitelboim Gravity, arXiv:1807.06575 [INSPIRE].

[27] A. Castro, F. Larsen and I. Papadimitriou, $5 D$ rotating black holes and the $n A d S_{2} / n C F T_{1}$ correspondence, JHEP 10 (2018) 042 [arXiv: 1807.06988] [INSPIRE].

[28] N. Callebaut and H. Verlinde, Entanglement dynamics in $2 D$ CFT with boundary: entropic origin of JT gravity and Schwarzian QM, JHEP 05 (2019) 045 [arXiv:1808.05583] [INSPIRE].

[29] A. Kitaev and S.J. Suh, Statistical mechanics of a two-dimensional black hole, JHEP 05 (2019) 198 [arXiv: 1808.07032] [inSPIRE].

[30] U. Moitra, S.P. Trivedi and V. Vishal, Extremal and near-extremal black holes and

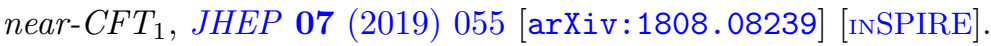

[31] Z. Yang, The quantum gravity dynamics of near extremal black holes, JHEP 05 (2019) 205 [arXiv: 1809.08647] [INSPIRE].

[32] A.R. Brown et al., Complexity of Jackiw-Teitelboim gravity, Phys. Rev. D 99 (2019) 046016 [arXiv: 1810.08741] [INSPIRE].

[33] F. Larsen and Y. Zeng, Black hole spectroscopy and AdS 2 holography, JHEP 04 (2019) 164 [arXiv: 1811.01288] [INSPIRE].

[34] A. Blommaert, T.G. Mertens and H. Verschelde, Fine structure of Jackiw-Teitelboim quantum gravity, JHEP 09 (2019) 066 [arXiv:1812.00918] [INSPIRE].

[35] M. Alishahiha, On complexity of Jackiw-Teitelboim gravity, Eur. Phys. J. C 79 (2019) 365 [arXiv:1811.09028] [INSPIRE].

[36] A. Dhar et al., Gravitational collapse in SYK models and Choptuik-like phenomenon, JHEP 11 (2019) 067 [arXiv: 1812.03979] [INSPIRE].

[37] K.S. Kolekar and K. Narayan, On AdS $S_{2}$ holography from redux, renormalization group flows and c-functions, JHEP 02 (2019) 039 [arXiv:1810.12528] [INSPIRE].

[38] K. Goto et al., Holographic complexity equals which action?, JHEP 02 (2019) 160 [arXiv: 1901.00014] [INSPIRE].

[39] S. Sachdev, Universal low temperature theory of charged black holes with $A d S_{2}$ horizons, J. Math. Phys. 60 (2019) 052303 [arXiv: 1902.04078] [INSPIRE].

[40] A. Blommaert, T.G. Mertens and H. Verschelde, Clocks and rods in Jackiw-Teitelboim quantum gravity, JHEP 09 (2019) 060 [arXiv:1902.11194] [INSPIRE].

[41] P. Saad, S.H. Shenker and D. Stanford, JT gravity as a matrix integral, arXiv:1903.11115 [INSPIRE].

[42] T.G. Mertens, Towards black hole evaporation in Jackiw-Teitelboim gravity, JHEP 07 (2019) 097 [arXiv: 1903.10485] [INSPIRE].

[43] J. Maldacena, G.J. Turiaci and Z. Yang, Two dimensional nearly de Sitter gravity, arXiv:1904.01911 [INSPIRE]. 
[44] T.G. Mertens and G.J. Turiaci, Defects in Jackiw-Teitelboim quantum gravity, JHEP 08 (2019) 127 [arXiv:1904.05228] [INSPIRE].

[45] L.V. Iliesiu, S.S. Pufu, H. Verlinde and Y. Wang, An exact quantization of Jackiw-Teitelboim gravity, JHEP 11 (2019) 091 [arXiv: 1905.02726] [INSPIRE].

[46] H.W. Lin, J. Maldacena and Y. Zhao, Symmetries near the horizon, JHEP 08 (2019) 049 [arXiv: 1904.12820] [INSPIRE].

[47] J. Cotler, K. Jensen and A. Maloney, Low-dimensional de Sitter quantum gravity, arXiv: 1905.03780 [INSPIRE].

[48] U. Moitra, S.K. Sake, S.P. Trivedi and V. Vishal, Jackiw-Teitelboim gravity and rotating black holes, JHEP 11 (2019) 047 [arXiv: 1905.10378] [INSPIRE].

[49] A. Castro and V. Godet, Breaking away from the near horizon of extreme Kerr, arXiv: 1906.09083 [INSPIRE].

[50] D. Stanford and E. Witten, JT gravity and the ensembles of random matrix theory, arXiv: 1907.03363 [INSPIRE].

[51] D.J. Gross, J. Kruthoff, A. Rolph and E. Shaghoulian, $T \bar{T}$ in $A d S_{2}$ and Quantum Mechanics, Phys. Rev. D 101 (2020) 026011 [arXiv:1907.04873] [INSPIRE].

[52] J. Hong, F. Larsen and J.T. Liu, The scales of black holes with nAdS $S_{2}$ geometry, JHEP 10 (2019) 260 [arXiv: 1907.08862] [INSPIRE].

[53] G. Sárosi, AdS $S_{2}$ holography and the SYK model, PoS Modave2017 (2018) 001 [arXiv: 1711.08482] [INSPIRE].

[54] L.K. Joshi, A. Mukhopadhyay and A. Soloviev, Time-dependent $N$ AdS $S_{2}$ holography with applications, Phys. Rev. D 101 (2020) 066001 [arXiv:1901.08877] [INSPIRE].

[55] S. Sachdev and J. Ye, Gapless spin fluid ground state in a random, quantum Heisenberg magnet, Phys. Rev. Lett. 70 (1993) 3339 [cond-mat/9212030] [INSPIRE].

[56] A. Kitaev, A simple model of quantum holography, talks given at KITP, April 7 and May 27 (2015).

[57] J. Polchinski and V. Rosenhaus, The spectrum in the Sachdev-Ye-Kitaev model, JHEP 04 (2016) 001 [arXiv: 1601.06768] [INSPIRE].

[58] J. Maldacena and D. Stanford, Remarks on the Sachdev-Ye-Kitaev model, Phys. Rev. D 94 (2016) 106002 [arXiv:1604.07818] [InSPIRE].

[59] A. Jevicki, K. Suzuki and J. Yoon, Bi-local holography in the SYK model, JHEP 07 (2016) 007 [arXiv: 1603.06246] [INSPIRE].

[60] I. Danshita, M. Hanada and M. Tezuka, Creating and probing the Sachdev-Ye-Kitaev model with ultracold gases: towards experimental studies of quantum gravity, PTEP 2017 (2017) 083I01 [arXiv: 1606.02454] [INSPIRE].

[61] D. Bagrets, A. Altland and A. Kamenev, Sachdev-Ye-Kitaev model as Liouville quantum mechanics, Nucl. Phys. B 911 (2016) 191 [arXiv:1607.00694] [INSPIRE].

[62] A. Jevicki and K. Suzuki, Bi-local holography in the SYK model: perturbations, JHEP 11 (2016) 046 [arXiv: 1608.07567] [INSPIRE].

[63] Y. Gu, X.-L. Qi and D. Stanford, Local criticality, diffusion and chaos in generalized Sachdev-Ye-Kitaev models, JHEP 05 (2017) 125 [arXiv:1609.07832] [INSPIRE].

[64] D.J. Gross and V. Rosenhaus, A generalization of Sachdev-Ye-Kitaev, JHEP 02 (2017) 093 [arXiv: 1610.01569] [InSPIRE]. 
[65] M. Berkooz, P. Narayan, M. Rozali and J. Simón, Higher dimensional generalizations of the SYK model, JHEP 01 (2017) 138 [arXiv:1610.02422] [INSPIRE].

[66] A.M. García-García and J.J.M. Verbaarschot, Spectral and thermodynamic properties of the Sachdev-Ye-Kitaev model, Phys. Rev. D 94 (2016) 126010 [arXiv:1610.03816] [InSPIRE].

[67] W. Fu, D. Gaiotto, J. Maldacena and S. Sachdev, Supersymmetric Sachdev-Ye-Kitaev models, Phys. Rev. D 95 (2017) 026009 [arXiv: 1610.08917] [INSPIRE].

[68] E. Witten, An SYK-like model without disorder, J. Phys. A 52 (2019) 474002 [arXiv: 1610.09758] [INSPIRE].

[69] R. Gurau, The complete $1 / N$ expansion of a SYK-like tensor model, Nucl. Phys. B 916 (2017) 386 [arXiv: 1611.04032] [INSPIRE].

[70] I.R. Klebanov and G. Tarnopolsky, Uncolored random tensors, melon diagrams and the Sachdev-Ye-Kitaev models, Phys. Rev. D 95 (2017) 046004 [arXiv:1611.08915] [InSPIRE].

[71] R.A. Davison et al., Thermoelectric transport in disordered metals without quasiparticles: The Sachdev-Ye-Kitaev models and holography, Phys. Rev. B 95 (2017) 155131 [arXiv: 1612.00849] [INSPIRE].

[72] C. Peng, M. Spradlin and A. Volovich, A supersymmetric SYK-like tensor model, JHEP 05 (2017) 062 [arXiv: 1612.03851] [InSPIRE].

[73] C. Krishnan, S. Sanyal and P.N. Bala Subramanian, Quantum chaos and holographic tensor models, JHEP 03 (2017) 056 [arXiv:1612.06330] [INSPIRE].

[74] G. Turiaci and H. Verlinde, Towards a 2d QFT analog of the SYK model, JHEP 10 (2017) 167 [arXiv: 1701.00528] [INSPIRE].

[75] T. Li, J. Liu, Y. Xin and Y. Zhou, Supersymmetric SYK model and random matrix theory, JHEP 06 (2017) 111 [arXiv:1702.01738] [INSPIRE].

[76] R. Gurau, Quenched equals annealed at leading order in the colored SYK model, EPL 119 (2017) 30003 [arXiv: 1702.04228] [inSPIRE].

[77] G. Mandal, P. Nayak and S.R. Wadia, Coadjoint orbit action of Virasoro group and two-dimensional quantum gravity dual to SYK/tensor models, JHEP 11 (2017) 046 [arXiv: 1702.04266] [INSPIRE].

[78] V. Bonzom, L. Lionni and A. Tanasa, Diagrammatics of a colored SYK model and of an SYK-like tensor model, leading and next-to-leading orders, J. Math. Phys. 58 (2017) 052301 [arXiv: 1702.06944] [INSPIRE].

[79] D.J. Gross and V. Rosenhaus, The bulk dual of SYK: cubic couplings, JHEP 05 (2017) 092 [arXiv: 1702.08016] [INSPIRE].

[80] D. Stanford and E. Witten, Fermionic localization of the Schwarzian theory, JHEP 10 (2017) 008 [arXiv: 1703.04612] [inSPIRE].

[81] C. Krishnan, K.V.P. Kumar and S. Sanyal, Random matrices and holographic tensor models, JHEP 06 (2017) 036 [arXiv: 1703.08155] [INSPIRE].

[82] S.R. Das, A. Jevicki and K. Suzuki, Three dimensional view of the SYK/AdS duality, JHEP 09 (2017) 017 [arXiv: 1704.07208] [INSPIRE].

[83] P. Narayan and J. Yoon, SYK-like tensor models on the lattice, JHEP 08 (2017) 083 [arXiv: 1705. 01554] [INSPIRE].

[84] S. Chaudhuri et al., Abelian tensor models on the lattice, Phys. Rev. D 97 (2018) 086007 [arXiv: 1705.01930] [INSPIRE]. 
[85] J. Murugan, D. Stanford and E. Witten, More on supersymmetric and $2 d$ analogs of the SYK model, JHEP 08 (2017) 146 [arXiv:1706. 05362] [INSPIRE].

[86] C. Krishnan and K.V.P. Kumar, Towards a finite-N hologram, JHEP 10 (2017) 099 [arXiv: 1706. 05364] [INSPIRE].

[87] D.J. Gross and V. Rosenhaus, A line of CFTs: from generalized free fields to SYK, JHEP 07 (2017) 086 [arXiv:1706.07015] [INSPIRE].

[88] A.M. García-García, B. Loureiro, A. Romero-Bermúdez and M. Tezuka, Chaotic-Integrable Transition in the Sachdev-Ye-Kitaev Model, Phys. Rev. Lett. 120 (2018) 241603 [arXiv: 1707.02197] [INSPIRE].

[89] D. Anninos, T. Anous and R.T. D'Agnolo, Marginal deformations \&3 rotating horizons, JHEP 12 (2017) 095 [arXiv: 1707.03380] [INSPIRE].

[90] S. Giombi, I.R. Klebanov and G. Tarnopolsky, Bosonic tensor models at large $N$ and small є, Phys. Rev. D 96 (2017) 106014 [arXiv:1707.03866] [InSPIRE].

[91] J. Sonner and M. Vielma, Eigenstate thermalization in the Sachdev-Ye-Kitaev model, JHEP 11 (2017) 149 [arXiv: 1707.08013] [INSPIRE].

[92] K. Bulycheva, I.R. Klebanov, A. Milekhin and G. Tarnopolsky, Spectra of operators in large $N$ tensor models, Phys. Rev. D 97 (2018) 026016 [arXiv:1707.09347] [INSPIRE].

[93] S. Choudhury et al., Notes on melonic $O(N)^{q-1}$ tensor models, JHEP 06 (2018) 094 [arXiv: 1707.09352] [INSPIRE].

[94] D.J. Gross and V. Rosenhaus, All point correlation functions in SYK, JHEP 12 (2017) 148 [arXiv: 1710.08113] [INSPIRE].

[95] A. Kitaev and S.J. Suh, The soft mode in the Sachdev-Ye-Kitaev model and its gravity dual, JHEP 05 (2018) 183 [arXiv:1711.08467] [INSPIRE].

[96] S.R. Das, A. Ghosh, A. Jevicki and K. Suzuki, Three dimensional view of arbitrary q SYK models, JHEP 02 (2018) 162 [arXiv:1711.09839] [INSPIRE].

[97] S.R. Das, A. Ghosh, A. Jevicki and K. Suzuki, Space-time in the SYK model, JHEP 07 (2018) 184 [arXiv: 1712.02725] [INSPIRE].

[98] P. Narayan and J. Yoon, Supersymmetric SYK Model with global symmetry, JHEP 08 (2018) 159 [arXiv: 1712. 02647] [inSPIRE].

[99] F.M. Haehl and M. Rozali, Fine grained chaos in AdS $S_{2}$ gravity, Phys. Rev. Lett. 120 (2018) 121601 [arXiv: 1712.04963] [InSPIRE].

[100] A.M. García-García and M. Tezuka, Many-body localization in a finite-range Sachdev-Ye-Kitaev model and holography, Phys. Rev. B 99 (2019) 054202 [arXiv: 1801.03204] [INSPIRE].

[101] C. Krishnan and K.V. Pavan Kumar, Exact solution of a strongly coupled gauge theory in $0+1$ dimensions, Phys. Rev. Lett. 120 (2018) 201603 [arXiv:1802.02502] [INSPIRE].

[102] D.A. Roberts, D. Stanford and A. Streicher, Operator growth in the SYK model, JHEP 06 (2018) 122 [arXiv: 1802.02633] [InSPIRE].

[103] D. Benedetti and R. Gurau, 2PI effective action for the SYK model and tensor field theories, JHEP 05 (2018) 156 [arXiv:1802.05500] [INSPIRE].

[104] I.R. Klebanov, A. Milekhin, F. Popov and G. Tarnopolsky, Spectra of eigenstates in fermionic tensor quantum mechanics, Phys. Rev. D 97 (2018) 106023 [arXiv:1802.10263] [INSPIRE]. 
[105] H. Gharibyan, M. Hanada, S.H. Shenker and M. Tezuka, Onset of random matrix behavior in scrambling systems, JHEP 07 (2018) 124 [Erratum ibid. 02 (2019) 197] [arXiv: 1803.08050] [INSPIRE].

[106] P. Saad, S.H. Shenker and D. Stanford, A semiclassical ramp in SYK and in gravity, arXiv: 1806.06840 [INSPIRE].

[107] S.S. Gubser, C. Jepsen, Z. Ji and B. Trundy, Higher melonic theories, JHEP 09 (2018) 049 [arXiv: 1806.04800] [INSPIRE].

[108] A. Blommaert, T.G. Mertens and H. Verschelde, The Schwarzian theory - A Wilson line perspective, JHEP 12 (2018) 022 [arXiv:1806.07765] [INSPIRE].

[109] C.-M. Chang, S. Colin-Ellerin and M. Rangamani, On melonic supertensor models, JHEP 10 (2018) 157 [arXiv: 1806.09903] [INSPIRE].

[110] G. Gur-Ari, R. Mahajan and A. Vaezi, Does the SYK model have a spin glass phase?, JHEP 11 (2018) 070 [arXiv:1806.10145] [INSPIRE].

[111] J. Liu, E. Perlmutter, V. Rosenhaus and D. Simmons-Duffin, d-dimensional SYK, AdS loops and $6 j$ symbols, JHEP 03 (2019) 052 [arXiv:1808.00612] [INSPIRE].

[112] S. Giombi et al., Prismatic large $N$ models for bosonic tensors, Phys. Rev. D 98 (2018) 105005 [arXiv: 1808.04344] [InSPIRE].

[113] K. Pakrouski, I.R. Klebanov, F. Popov and G. Tarnopolsky, Spectrum of majorana quantum mechanics with $O(4)^{3}$ symmetry, Phys. Rev. Lett. 122 (2019) 011601 [arXiv:1808.07455] [INSPIRE].

[114] M. Blake, R.A. Davison, S. Grozdanov and H. Liu, Many-body chaos and energy dynamics in holography, JHEP 10 (2018) 035 [arXiv: 1809.01169] [INSPIRE].

[115] R. Bhattacharya, D.P. Jatkar and N. Sorokhaibam, Quantum quenches and thermalization in SYK models, JHEP 07 (2019) 066 [arXiv:1811.06006] [INSPIRE].

[116] J. Murugan and H. Nastase, One-dimensional bosonization and the SYK model, JHEP 08 (2019) 117 [arXiv: 1812.11929] [InSPIRE].

[117] J. Kim, I.R. Klebanov, G. Tarnopolsky and W. Zhao, Symmetry breaking in coupled SYK or tensor models, Phys. Rev. X 9 (2019) 021043 [arXiv: 1902.02287] [INSPIRE].

[118] F. Sun, Y. Yi-Xiang, J. Ye and W.M. Liu, Classification of the quantum chaos in colored Sachdev-Ye-Kitaev models, Phys. Rev. D 101 (2020) 026009 [arXiv: 1903. 02213] [INSPIRE].

[119] H. Guo, Y. Gu and S. Sachdev, Transport and chaos in lattice Sachdev-Ye-Kitaev models, Phys. Rev. B 100 (2019) 045140 [arXiv: 1904.02174] [INSPIRE].

[120] P. Nayak, J. Sonner and M. Vielma, Eigenstate thermalisation in the conformal Sachdev-Ye-Kitaev model: an analytic approach, JHEP 10 (2019) 019 [arXiv:1903.00478] [INSPIRE].

[121] F. Sun and J. Ye, Periodic table of SYK and supersymmetric SYK, arXiv:1905.07694 [INSPIRE].

[122] I.R. Klebanov, P.N. Pallegar and F.K. Popov, Majorana fermion quantum mechanics for higher rank tensors, Phys. Rev. D 100 (2019) 086003 [arXiv: 1905.06264] [INSPIRE].

[123] V. Rosenhaus, An introduction to the SYK model, arXiv:1807.03334 [INSPIRE].

[124] Y. Chen and P. Zhang, Entanglement entropy of two coupled SYK models and eternal traversable wormhole, JHEP 07 (2019) 033 [arXiv: 1903.10532] [INSPIRE]. 
[125] A.C. Wall, Ten proofs of the generalized second law, JHEP 06 (2009) 021 [arXiv:0901.3865] [INSPIRE].

[126] A.C. Wall, Proving the achronal averaged null energy condition from the generalized second law, Phys. Rev. D 81 (2010) 024038 [arXiv:0910.5751] [inSPIRE].

[127] A.C. Wall, The generalized second law implies a quantum singularity theorem, Class. Quant. Grav. 30 (2013) 165003 [Erratum ibid. 30 (2013) 199501] [arXiv:1010.5513] [INSPIRE].

[128] A.C. Wall, A proof of the generalized second law for rapidly changing fields and arbitrary horizon slices, Phys. Rev. D 85 (2012) 104049 [arXiv:1105.3445] [INSPIRE].

[129] A.C. Wall, Testing the generalized second law in $1+1$ dimensional conformal vacua: an argument for the causal horizon, Phys. Rev. D 85 (2012) 024015 [arXiv:1105.3520] [INSPIRE].

[130] R. Bousso and N. Engelhardt, Proof of a new area law in general relativity, Phys. Rev. D 92 (2015) 044031 [arXiv: 1504.07660] [INSPIRE].

[131] R. Bousso and N. Engelhardt, New area law in general relativity, Phys. Rev. Lett. 115 (2015) 081301 [arXiv: 1504.07627] [inSPIRE].

[132] R. Bousso, Z. Fisher, S. Leichenauer and A.C. Wall, Quantum focusing conjecture, Phys. Rev. D 93 (2016) 064044 [arXiv: 1506. 02669] [INSPIRE].

[133] R. Bousso et al., Proof of the quantum null energy condition, Phys. Rev. D 93 (2016) 024017 [arXiv: 1509. 02542] [INSPIRE].

[134] R. Bousso and N. Engelhardt, Generalized second law for cosmology, Phys. Rev. D 93 (2016) 024025 [arXiv: 1510.02099] [inSPIRE].

[135] N. Engelhardt and A.C. Wall, Decoding the apparent horizon: coarse-grained holographic entropy, Phys. Rev. Lett. 121 (2018) 211301 [arXiv:1706.02038] [INSPIRE].

[136] A.C. Wall, A survey of black hole thermodynamics, arXiv:1804.10610 [INSPIRE].

[137] R. Bousso, Y. Nomura and G.N. Remmen, Outer entropy and quasilocal energy, Phys. Rev. D 99 (2019) 046002 [arXiv: 1812.06987] [INSPIRE].

[138] T.M. Fiola, J. Preskill, A. Strominger and S.P. Trivedi, Black hole thermodynamics and information loss in two-dimensions, Phys. Rev. D 50 (1994) 3987 [hep-th/9403137] [INSPIRE].

[139] C. Holzhey, F. Larsen and F. Wilczek, Geometric and renormalized entropy in conformal field theory, Nucl. Phys. B 424 (1994) 443 [hep-th/9403108] [InSPIRE].

[140] G. 't Hooft, On the quantum structure of a black hole, Nucl. Phys. B 256 (1985) 727.

[141] L. Susskind, Some speculations about black hole entropy in string theory, hep-th/9309145 [INSPIRE].

[142] L. Susskind and J. Uglum, Black hole entropy in canonical quantum gravity and superstring theory, Phys. Rev. D 50 (1994) 2700 [hep-th/9401070] [INSPIRE].

[143] N. Engelhardt and A.C. Wall, Quantum extremal surfaces: holographic entanglement entropy beyond the classical regime, JHEP 01 (2015) 073 [arXiv: 1408.3203] [INSPIRE].

[144] S.P. Trivedi, Semiclassical extremal black holes, Phys. Rev. D 47 (1993) 4233 [hep-th/9211011] [INSPIRE].

[145] G. Penington, Entanglement wedge reconstruction and the information paradox, arXiv:1905.08255 [INSPIRE]. 
[146] A. Almheiri, N. Engelhardt, D. Marolf and H. Maxfield, The entropy of bulk quantum fields and the entanglement wedge of an evaporating black hole, JHEP 12 (2019) 063 [arXiv: 1905. 08762] [INSPIRE].

[147] A. Almheiri, R. Mahajan, J. Maldacena and Y. Zhao, to appear.

[148] P.C.W. Davies, S.A. Fulling and W.G. Unruh, Energy momentum tensor near an evaporating black hole, Phys. Rev. D 13 (1976) 2720 [InSPIRE].

[149] S.M. Christensen and S.A. Fulling, Trace anomalies and the Hawking effect, Phys. Rev. D 15 (1977) 2088 [INSPIRE].

[150] N.D. Birrell and P.C.W. Davies, Quantum fields in curved space, Cambridge Monographs on Mathematical Physics, Cambridge University Press, Cambridge U.K. (1984).

[151] M. Henningson and K. Skenderis, The holographic Weyl anomaly, JHEP 07 (1998) 023 [hep-th/9806087] [INSPIRE].

[152] V. Balasubramanian and P. Kraus, A stress tensor for Anti-de Sitter gravity, Commun. Math. Phys. 208 (1999) 413 [hep-th/9902121] [InSPIRE].

[153] R.C. Myers, Stress tensors and Casimir energies in the AdS/CFT correspondence, Phys. Rev. D 60 (1999) 046002 [hep-th/9903203] [INSPIRE].

[154] R. Emparan, C.V. Johnson and R.C. Myers, Surface terms as counterterms in the AdS/CFT correspondence, Phys. Rev. D 60 (1999) 104001 [hep-th/9903238] [INSPIRE].

[155] R.B. Mann, Entropy of rotating Misner string space-times, Phys. Rev. D 61 (2000) 084013 [hep-th/9904148] [INSPIRE].

[156] P. Kraus, F. Larsen and R. Siebelink, The gravitational action in asymptotically AdS and flat space-times, Nucl. Phys. B 563 (1999) 259 [hep-th/9906127] [INSPIRE].

[157] S. de Haro, S.N. Solodukhin and K. Skenderis, Holographic reconstruction of space-time and renormalization in the AdS/CFT correspondence, Commun. Math. Phys. 217 (2001) 595 [hep-th/0002230] [INSPIRE].

[158] M. Bianchi, D.Z. Freedman and K. Skenderis, Holographic renormalization, Nucl. Phys. B 631 (2002) 159 [hep-th/0112119] [INSPIRE].

[159] K. Skenderis, Lecture notes on holographic renormalization, Class. Quant. Grav. 19 (2002) 5849 [hep-th/0209067] [INSPIRE].

[160] J. Polchinski, String theory. Volume 1: an introduction to the bosonic string, Cambridge Monographs on Mathematical Physics, Cambridge University Press, Cambridge U.K. (2007).

[161] C.P. Herzog, K.-W. Huang and K. Jensen, Universal entanglement and boundary geometry in conformal field theory, JHEP 01 (2016) 162 [arXiv: 1510.00021] [INSPIRE]. 